\title{
Path integral quantization of a spinning particle
}

\author{
Jerzy Kowalski-Glikman๑* \\ Institute for Theoretical Physics, University of Wrocław, pl. M. Borna 9, 50-204 Wrocław, Poland \\ and National Centre for Nuclear Research, Pasteura 7, 02-093 Warsaw, Poland \\ Giacomo Rosati $\oplus^{\dagger}$ \\ Institute for Theoretical Physics, University of Wrocław, pl. M. Borna 9, 50-204 Wrocław, Poland
}

(Received 14 January 2020; accepted 20 February 2020; published 12 March 2020)

\begin{abstract}
Following the idea of Alekseev and Shatashvili, we derive the path integral quantization of a modified relativistic particle action that results in the Feynman propagator of a free field with arbitrary spin. This propagator can be associated with the Duffin, Kemmer, and Petiau (DKP) form of a free field theory. We show explicitly that the obtained DKP propagator is equivalent to the standard one, for spins 0 and 1. We argue that this equivalence holds also for higher spins.
\end{abstract}

DOI: 10.1103/PhysRevD.101.065003

\section{INTRODUCTION}

Spin is a subtle and elusive concept. On the one hand, one would think that it should be very easy to describe. Indeed, like the momentum carried by a particle is an eigenvalue of the translation operator, spin can be described as an eigenvalue of the operators associated with rotations (usually taken to be the $z$-component of angular momentum and the angular momentum squared). One would think that it is pretty easy then to find a description of a classical relativistic particle with spin and then to quantize it, so as to obtain the free theory of a field with spin. But this is not the case. There are many attempts to formulate a classical spin theory, both in terms of commuting and anticommuting classical variables, that after quantization produce the expected quantum fields with spin, reviewed, for example, in [1]. Among the commutative models, a particularly interesting one was formulated by Balachandran et al. [2,3], who assumed that the configuration space of a spinning particle should be identified with the Poincaré group. This construction was later found very fruitful, for example, in the case of particles coupled to gravity in three [4] and four [5] spacetime dimensions. Some other approaches are reviewed in the recent paper [6].

A particularly convenient approach to quantization is path integral. It is well known that when one uses the path

\footnotetext{
jerzy.kowalski-glikman@uwr.edu.pl

giacomo.rosati@uwr.edu.pl
}

Published by the American Physical Society under the terms of the Creative Commons Attribution 4.0 International license. Further distribution of this work must maintain attribution to the author(s) and the published article's title, journal citation, and DOI. Funded by SCOAP ${ }^{3}$. integral to describe the quantum transition amplitude of a relativistic particle, one gets as a result a scalar (Feynman) propagator of the form $\left(p^{2}-m^{2}+i \epsilon\right)^{-1}$. This is perfectly consistent with the result of canonical quantization, in which case the classical first-class constraint $p^{2}-m^{2}=0$ becomes, after quantization, according to Dirac procedure, the Klein-Gordon equation $\left(\square+m^{2}\right) \phi=0$.

The question arises if one can find the "spinning" relativistic particle action such that, after plugging it into the path integral, one gets as a result the Dirac propagator $(\not p-m+i \epsilon)^{-1}$ in the case of spin-1/2 and appropriate expressions for higher spins. Clearly, this requires two kinds of modifications of the standard relativistic particle action. First, contrary to the scalar propagator, Dirac propagator has a term linear in momentum. Second, the path integral should produce the right representation of Dirac $\gamma$ matrices from some classical data. It is the aim of the present paper to describe such construction in details. In our approach, we follow the scheme proposed some time ago by Alekseev and Shatashvili ${ }^{1}$ [7], which, in turn, was motivated by the construction proposed by Polyakov in [9].

To this aim in Sec. II, after presenting the standard scalar path integral, we observe that the Dirac form of the propagator can be obtained if we start with a relativistic particle action in which the first-class constraint becomes linear in momenta. Parallel to that, we notice that an analogous approach can be phrased in terms of the Duffin, Kemmer, and Petiau (DKP) formalism for a field theory with spin-0 and spin-1. We first show how for a scalar (spin-0) field, the second order formalism can be naturally associated with the first order DKP formulation. In Sec. III,

\footnotetext{
${ }^{1}$ For a different approach leading to similar results as the ones in [7], see also [8].
} 
we construct the spinning particle path integral, obtaining the general expression for the propagator. This section relies on some more technical results, which are described in details in appendices. In Sec. IV, we complete the explicit construction of the propagator for spin- 0 , spin- $1 / 2$, and spin-1, and we prove that the so obtained DKP propagators are equivalent to the standard ones. We do not attempt to extend our construction to spins higher than 1 explicitly, although there are little doubts that such a generalization can be done. Unfortunately, the technical difficulty of the formalism grows rapidly with growing spin, as the equations defining the higher-spin analogs of Dirac $\gamma$ matrices are getting more and more complicated. Section V is devoted to conclusions and discussion of open problems.

\section{THE SCALAR PATH INTEGRAL}

Consider the standard, free, scalar relativistic particle moving in four-dimensional spacetime, between the spacetime point with coordinates $x_{1}$ and the one with coordinates $x_{2}$. The transition amplitude from the initial state $\langle$ in $|=$ $\left\langle x_{1}\right|$ to the final one $\mid$ out $\rangle=\left|x_{2}\right\rangle$ is given by the path integral $^{2}$ for the trajectories beginning at $x_{1}$ and ending in $x_{2}$,

$$
G\left(x_{2}, x_{1}\right)=\int_{x(0)=x_{1}}^{x(1)=x_{2}} \mathcal{D}(x(\tau)) \mathcal{D}(p(\tau)) \mathcal{D}(\mathcal{N}(\tau) / \text { Diff }) e^{i S}
$$

where the action $S$ is

$$
S=\int_{0}^{1} d \tau\left(p \dot{x}-\mathcal{N}\left(p^{2}-m^{2}\right)\right),
$$

and $\mathcal{D}(\mathcal{N}(\tau) /$ Diff $)$ denotes the measure on the Lagrange multiplier $\mathcal{N}$ up to worldline reparametrization, under which it transforms as a one-dimensional metric determinant.

Now, we integrate over $x(\tau)$. In order to do that, we must first rewrite it in the form that conveniently takes into account the boundary conditions,

$$
x(\tau)=x_{1}+\left(x_{2}-x_{1}\right) \tau+y(\tau), \quad y(0)=y(1)=0 .
$$

Clearly, $\mathcal{D}(x(\tau))=\mathcal{D}(y(\tau))$. Now, we can integrate by parts the action (2) and then integrate over $y(\tau)$ obtaining

$$
\begin{aligned}
G\left(x_{2}, x_{1}\right)= & \int \mathcal{D}(p(\tau)) \mathcal{D}(\mathcal{N}(\tau) / \operatorname{Diff}) \delta(\dot{p}) e^{i p\left(x_{2}-x_{1}\right)} \\
& \times \exp \left(-i\left(p^{2}-m^{2}\right) \int_{0}^{1} d \tau \mathcal{N}\right) .
\end{aligned}
$$

\footnotetext{
${ }^{2}$ In this paper, we denote the four-dimensional indices by $\mu=0,1,2,3$, raised and lowered by the 4D Minkowski metric $\eta_{\mu \nu}=\operatorname{diag}(1,-1,-1,-1) ; u \cdot v$ and $v^{2}$ are shorthand for $u^{\mu} v_{\mu}$ and $v_{\mu} v^{\mu}$, respectively, while $\boldsymbol{u} \cdot \boldsymbol{v}=\sum_{i=1}^{3} u_{i} v_{i}$.
}

Noticing now that $\delta(\dot{p})$ enforces the momenta to be $\tau$-independent, so that $\mathcal{D}(p(\tau)) \delta(\dot{p})=d^{4} p$, and that

$$
L \equiv \int_{0}^{1} d \tau \mathcal{N}>0
$$

is the gauge invariant information carried by $\mathcal{N}^{3}$; we can express the path integral (4) as

$$
\begin{aligned}
G\left(x_{2}, x_{1}\right) & =\int d^{4} p \int_{0}^{\infty} d L e^{i p\left(x_{2}-x_{1}\right)} e^{-i L\left(p^{2}-m^{2}-i \epsilon\right)} \\
& =\int d^{4} p \frac{i}{p^{2}-m^{2}-i \epsilon} e^{i p\left(x_{2}-x_{1}\right)}
\end{aligned}
$$

where we added the $-i \epsilon$ term to regularize the integral, as usual. The Fourier transform of the transition amplitude (5) is the (Feynman) propagator of the quantum scalar field.

The approach outlined above cannot be directly applied to the case of fields with higher spin. For example, in the case of spin- $1 / 2$ field, the propagator is the inverse of an expression linear in momentum, $(\not p-m)^{-1}$, instead of the inverse of quadratic expression, $\left(p^{2}-m^{2}\right)^{-1}$, as in (5). It was not long after Dirac's formulation of a theory of spin $1 / 2$-fields, when a similar (unified) formulation for fields of spin-0 and spin-1 was put forward by DKP [11-13]. While the details of the DKP theory needed for our analysis will be discussed in Sec. IV, let us present here a brief introduction to this approach for the scalar (spin-0) fields.

The very reason behind the $p^{2}-m^{2}$ term in the scalar field propagator is the form of scalar field equations that follow from the Lagrangian,

$$
L=\partial_{\mu} \phi \partial^{\mu} \phi-m^{2} \phi^{2}
$$

In order to get the DKP propagator, inverse proportional to momentum (instead of its square), we must rewrite the Lagrangian (6) in the form linear in spacetime derivatives, similar to the form of Dirac Lagrangian. This can be achieved by turning from the second order formulation (with second order derivatives) to the first order one, in which the field $\phi$ and its derivatives $\partial \phi$ are treated as independent field components of a multicomponent field,

$$
\psi=\left(\pi_{0}, \pi_{1}, \pi_{2}, \pi_{3}, \varphi\right)^{T} .
$$

For a real scalar field $\varphi^{\dagger}=\varphi$, the DKP Lagrangian takes the form, ${ }^{4}$

\footnotetext{
${ }^{3}$ It is essential at this point that $\mathcal{N}$, being the one-dimensional Euclidean metric, is positive. See [10] for the recent detailed discussion on this issue.

${ }^{4}$ Here, we adopt a specific representation for the $\beta$ matrices presented in Appendix D, and we refer to Sec. IV for details.
} 


$$
\begin{aligned}
L_{\mathrm{DKP}}= & \frac{i}{2} \bar{\psi} \beta^{\mu} \partial_{\mu} \psi-\frac{i}{2}\left(\partial_{\mu} \bar{\psi}\right) \beta^{\mu} \psi-m \bar{\psi} \psi \\
= & \frac{i}{2} \sum_{\mu}\left(\left(\pi_{\mu}^{\dagger}-\pi_{\mu}\right) \partial_{\mu} \varphi-\varphi \partial_{\mu}\left(\pi_{\mu}^{\dagger}-\pi_{\mu}\right)\right) \\
& -m \pi_{\mu}^{\dagger} \pi^{\mu}-m \varphi^{2},
\end{aligned}
$$

where $\beta^{\mu}$ are the so-called DKP $\beta$ matrices, playing, for the spin-0 and spin- 1 theories, a role analogous to that of Dirac $\gamma$ matrices for spin-1/2, and the adjoint field $\bar{\psi}$ are defined as

$$
\bar{\psi}=\psi^{\dagger} \eta_{0}=\left(\pi_{0}^{\dagger},-\pi_{1}^{\dagger},-\pi_{2}^{\dagger},-\pi_{3}^{\dagger}, \varphi^{\dagger}\right)
$$

with $\eta_{0} \equiv 2 \beta_{0}^{2}-\eta_{00} \rrbracket$.

Varying the DKP Lagrangian with respect to $\pi_{\mu}$ and $\pi_{\mu}^{\dagger}$, we obtain the expression for the conjugate momenta,

$$
\pi_{\mu}=\frac{i}{m} \eta^{\mu \nu} \partial_{\nu} \varphi, \quad \pi_{\mu}^{\dagger}=-\frac{i}{m} \eta^{\mu \nu} \partial_{\nu} \varphi,
$$

which, substituted into the DKP Lagrangian, gives back, after the identification $\varphi=\sqrt{m} \phi$, the quadratic Lagrangian (6). This shows that the two Lagrangians are equivalent (both classically and quantum mechanically), and one concludes that, for free fields, the DKP formalism is nothing but using the first order Lagrangian.

It follows that the DKP Lagrangian leads to the quantum propagator of the form

$$
G(p)=\frac{1}{\not p-m \mathbb{1}-i \epsilon}, \quad \not p=\beta_{\mu} p^{\mu} .
$$

It is expected that an analogous construction can be made for higher spins. In that case, the propagators for higher spins will have the same form, but with appropriately chosen matrices replacing the $\beta$ matrices of spin- $0 /$ spin- 1 theory.

A natural question arises as if it is possible to obtain this propagator from the path integral with some form of the particle action, as it was in the case of the scalar field above (5). The answer is positive, and in the next section we present the explicit construction.

\section{THE SPINNING PARTICLE PATH INTEGRAL}

In this section, we will discuss how the path integral for spinning particle can be written in the form proposed by Alekseev and Shatashvili [7], whose construction is, in turn, a generalization of the one of Polyakov [9]. We will omit the more technical aspects of the argument, presenting them in details in the appendix, stressing here the motivations and the meaning of the final result.

Our starting point is the path integral (4) in momentum representation,
$G(p)=\int \mathcal{D}(\mathcal{N}(\tau) /$ Diff $) \exp \left(-i \int_{0}^{1} d \tau \mathcal{N}\left(p^{2}-m^{2}\right)\right)$

Our goal is to generalize the form of (12) so as to make it describe a particle of an arbitrary spin.

We start with the observation that the action in (12) can be rewritten as

$\mathcal{N}\left(p^{2}-m^{2}\right)=m \mathcal{N}(p \cdot p / m-m)=\mathcal{N}^{\prime}(p v-m)$,

where we introduced a new variable $v$ that replaces $p / m$. The variable $v$ is, of course, nothing but the four velocity, satisfying $v^{2}=1$ and therefore belonging to the threedimensional pseudosphere $P S^{3}$.

Let us stop for a moment to contemplate on the meaning of (13). We replaced the second order constraint of the particle action $p^{2}-m^{2}=0$ with the first order one $p v-m=0$. The former leads to the standard scalar propagator (5), and it is natural to expect that the latter will lead to the DKP one (11) if we force the path integral to replace $v$ with the DKP matrices $\beta$. Now, the $\beta$ matrices, similarly to the Dirac matrices, are defined to satisfy (among others) the requirement that their commutator has the form

$$
\left[\beta_{\mu}, \beta_{\nu}\right]=S_{\mu \nu},
$$

where $S_{\mu \nu}$ generate Lorentz transformations [14] $U \simeq 1+$ $\frac{1}{2} \omega^{\mu \nu} S_{\mu \nu}$.

Since the commutator (14) must come as a result of quantization of a classical theory, the kinetic term (symplectic form) of the latter should be such that the associated Poisson bracket has the form

$$
\left\{v_{\mu}, v_{\nu}\right\}=j_{\mu \nu},
$$

where, again, $j$ are Lorentz generators, satisfying $\mathfrak{s} \mathfrak{o}(3,1)$ algebra. After quantization (as we will show below), the associated quantum operators satisfy the commutators,

$$
\left[\hat{v}_{\mu}, \hat{v}_{\nu}\right]=i \hat{j}_{\mu \nu},
$$

and one gets (14) after identifying

$$
\beta_{\mu} \equiv \hat{v}_{\mu}, \quad S_{\mu \nu} \equiv \hat{S}_{\mu \nu}=i \hat{j}_{\mu \nu}
$$

It turns out that in order to get the correct properties for the $\beta$ (as well as for the Dirac matrices $\gamma$, see, for instance, [15], Ch. 5.4), the operators $\hat{v}_{\mu}$ must be generators of Clifford algebra $C l_{3,1}$. Their operators $\hat{j}_{\mu \nu}$ generate the $\mathfrak{s} \mathfrak{o}(3,1)$ Lorentz algebra, and one can show that together $\hat{v}_{\mu}$ and $\hat{j}_{\mu \nu}$ generate the $\mathfrak{g} \mathfrak{o}(3,2)$ Lie algebra, the anti-de Sitter algebra. 
In turn, the matrices $\beta_{\mu}$ (or $\gamma_{\mu}$ ) and $S_{\mu \nu}$, obtained by the substitution (17), generate the $\mathfrak{\mathfrak { s }}(4,1)$ de Sitter algebra.

Now, since in the Poisson-Lie theory, there is a one-toone correspondence between commutators of the algebra and the Poisson structure on the dual algebra, it is natural to identify $v$ with elements of the Lie algebra $\mathfrak{S o}(3,2)^{*}$ "dual" to the one spanned by the generators of $\mathfrak{g} \mathfrak{o}(3,2)$. Let us discuss in details how this comes about.

We start from the $\mathfrak{g} \mathfrak{o}(3,2)$ Lie algebra, generated by the antisymmetric matrices $(A, B=0,1,2,3,4)$,

$$
\begin{aligned}
\left(M_{A B}\right)^{C}{ }_{D} & =-\left(M_{B A}\right)^{C}{ }_{D}=\delta_{A}^{C} \eta_{B E}-\delta_{B}^{C} \eta_{A E}, \\
\eta_{A B} & =\operatorname{diag}(1,-1,-1,-1,1)
\end{aligned}
$$

and defined by the Lie brackets

$$
\left[M_{A B}, M_{C D}\right]=\eta_{A D} M_{B C}+\eta_{B C} M_{A D}-\eta_{A C} M_{B D}-\eta_{B D} M_{A C} .
$$

With the redefinition $\Upsilon_{\mu}=M_{\mu 4}, J_{\mu \nu}=M_{\mu \nu}(\mu, \nu=0$, $1,2,3)$, the $\mathfrak{g} \mathfrak{v}(3,2)$ algebra takes the form

$$
\begin{aligned}
{\left[\Upsilon_{\mu}, \Upsilon_{\nu}\right] } & =J_{\mu \nu}, \quad\left[J_{\mu \nu}, \Upsilon_{\rho}\right]=\Upsilon_{\nu} \eta_{\mu \rho}-\Upsilon_{\mu} \eta_{\nu \rho}, \\
{\left[J_{\mu \nu}, J_{\rho \sigma}\right] } & =\eta_{\mu \rho} J_{\nu \sigma}+\eta_{\nu \sigma} J_{\mu \rho}-\eta_{\mu \sigma} J_{\nu \rho}-\eta_{\nu \rho} J_{\mu \sigma} .
\end{aligned}
$$

An arbitrary element $X$ of the dual algebra $\mathfrak{g} \mathfrak{o}(3,2)^{*}$ is spanned by the generators $\left\{\tilde{\Upsilon}^{\mu}, \tilde{J}^{\mu \nu}\right\}\left(\tilde{J}^{\nu \mu}=-\tilde{j}^{\mu \nu}\right)$, dual to $\left\{\Upsilon_{\mu}, J_{\mu \nu}\right\}$ in the sense that (see Appendix A) $\left\langle\tilde{\Upsilon}^{\mu}, \Upsilon_{\nu}\right\rangle=\delta_{\nu}^{\mu}$, $\left\langle\tilde{J}^{\mu \nu}, J_{\rho \sigma}\right\rangle=\delta_{\rho}^{\mu} \delta_{\sigma}^{\nu}-\delta_{\rho}^{\nu} \delta_{\sigma}^{\mu},\left\langle\tilde{\Upsilon}^{\mu}, J_{\rho \sigma}\right\rangle=\left\langle\tilde{J}^{\mu \nu}, \Upsilon_{\nu}\right\rangle=0$, and it has the form $\left(j_{\nu \mu}=-j_{\mu \nu}\right)$

$$
X=v_{\mu} \tilde{\Upsilon}^{\mu}+\frac{1}{2} j_{\mu \nu} \tilde{J}^{\mu \nu}
$$

Using the definitions presented in Appendix A, one can check that the coadjoint orbit of $v \equiv v_{\mu} \tilde{\Upsilon}^{\mu}$ under the action of the Lorentz subgroup is exactly the pseudosphere $P S^{3}$. The orbits are characterized by the values $\left(c_{1}, c_{2}\right)$ of the two polynomials of $v_{\mu}, j_{\mu \nu}$ invariant under the coadjoint action of $\mathrm{SO}(3,2)$, corresponding to the two Casimirs of $\mathfrak{s} \mathfrak{o}(3,2)$,

$$
\begin{aligned}
& \mathcal{C}_{1}=v^{\mu} v_{\mu}+\frac{1}{2} j_{\mu \nu} j^{\mu \nu}, \\
& \mathcal{C}_{2}=W_{0}^{2}-W \cdot W+\frac{1}{4}\left(\epsilon_{i j k} j_{j k} j_{i 0}\right)^{2},
\end{aligned}
$$

with

$W_{0}=\frac{1}{2} \epsilon_{i j k} j_{j k} v_{i}, \quad W=-\frac{1}{2} \epsilon_{i j k} j_{j k} v_{0}+\epsilon_{i j k} j_{j 0} v_{k}$.

The action in the path integral should therefore consist of two pieces. The first is given by (13), and the second is an action $S$ to be defined so as to impose the condition (15), and which leads to its quantization. It is given by [16]

$$
S=\int \omega
$$

where $\omega=\left\langle X(g), d g g^{-1}\right\rangle, g$ being an element of $\mathrm{SO}(3,2)$, is the Liouville form associated with Kirillov symplectic two form, discussed in details in Appendix A. This action leads to the following expressions for the Poisson brackets of the dynamical variables:

$$
\begin{aligned}
\left\{v_{\mu}, v_{\nu}\right\} & =j_{\mu \nu}, \quad\left\{j_{\mu \nu}, v_{\rho}\right\}=v_{\nu} \eta_{\mu \rho}-v_{\mu} \eta_{\nu \rho}, \\
\left\{j_{\mu \nu}, j_{\rho \sigma}\right\} & =\eta_{\mu \rho} j_{\nu \sigma}+\eta_{\nu \sigma} j_{\mu \rho}-\eta_{\mu \sigma} j_{\nu \rho}-\eta_{\nu \rho} j_{\mu \sigma} .
\end{aligned}
$$

This is exactly what we want, because after quantization the first equation above will become the defining equation for the $\beta$ matrices of the DKP formalism.

The final form of the momentum space propagator is therefore

$$
\begin{gathered}
G(p)=\int_{0}^{\infty} d L \int \mathcal{D}(\Upsilon(t), J(t)) \\
\times \exp \left(i m L-i \int_{0}^{L} d t p \cdot v(t)\right) \exp (i S(v, j)), \\
S(v, j)=\int_{0}^{L} d t \omega(v(t), j(t)) .
\end{gathered}
$$

It is shown in the Appendix B that the term $\exp (i S(v, j))$ "quantizes" the values of the invariant polynomials in $\left\{v_{\mu}, j_{\mu \nu}\right\}$ defining the orbits, so that the corresponding operators $\left\{\hat{v}_{\mu}, \hat{j}_{\mu \nu}\right\}$ belong to an irreducible representation $\left\{\pi\left(\hat{v}_{\mu}\right), \pi\left(\hat{j}_{\mu \nu}\right)\right\}$ of $\mathfrak{g} \mathfrak{v}(3,2)$ (or, through the substitution $\hat{S}_{\mu \nu}=i \hat{j}_{\mu \nu}$, to an irreducible representation $\left\{\pi\left(\hat{v}_{\mu}\right), \pi\left(\hat{S}_{\mu \nu}\right)\right\}$ of $\mathfrak{s} \mathfrak{o}(4,1)$ ). In other words, the path integral in (26) computes, for given boundary conditions, the correlation function

$$
\begin{aligned}
& \int \mathcal{D}(v(t), j(t)) \exp \left(i m L-i \int_{0}^{L} d t p \cdot v(t)\right) \\
& \quad \times \exp (i S(v, j))=\left\langle i\left|\exp \left(i \int_{0}^{L} p \cdot \hat{v} d t\right)\right| j\right\rangle
\end{aligned}
$$

between states $|i\rangle$ belonging to a particular representation of the $\mathfrak{s} \mathfrak{v}(4,1)$ algebra, corresponding to the particular choice of integral orbit. In the formula (28), $\hat{v}_{\mu}$ is the quantum operator corresponding to $v_{\mu}$, and, depending on the spin representation $|i\rangle$, it is given by a Dirac $\gamma$ matrix (for spin-1/2) or a DKP $\beta$ matrix for spin-0 or spin-1, and, presumably, to matrix representations for higher spins. 
In particular, these matrices must satisfy (14), and we will show now that this is indeed the case.

Let us denote by $l_{a} \equiv\left\{v_{\mu}, j_{\mu \nu}\right\}$ the coordinates on the dual Lie algebra $\mathfrak{g} \mathfrak{o}(3,2)^{*}$, so that the Poisson brackets (25) can be written concisely in terms of the $\mathfrak{g} \mathfrak{o}(3,2)$ structure constant $f_{a b}{ }^{c}$ defined by (20) as $\left\{l_{a}, l_{b}\right\}=f_{a b}{ }^{c}$. The transformation

$$
l_{a} \rightarrow l_{a}+f_{a b}^{c} \xi^{b} l_{c}
$$

is a symmetry of the classical action, and the resulting Ward identity reads (see Appendix $\mathrm{C}$ for details)

$$
\begin{aligned}
& \partial_{t}\left\langle i\left|\hat{l}_{a}(t) \hat{l}_{a_{1}}\left(t_{1}\right) \cdots \hat{l}_{a_{n}}\left(t_{n}\right)\right| j\right\rangle \\
& =i \sum_{k} f_{a a_{k}}{ }^{b}\left\langle i\left|\hat{l}_{b}(t) \hat{l}_{a_{1}}\left(t_{1}\right) \cdots \hat{t}_{a_{k}}\left(t_{k}\right) \cdots \hat{l}_{a_{n}}\left(t_{n}\right)\right| j\right\rangle \delta\left(t-t_{k}\right),
\end{aligned}
$$

where $\hat{t}_{a_{k}}\left(t_{k}\right)$ indicates that the particular term is missing.

In order to derive the equal time commutators (ETC) for the corresponding field operators, we can apply the Bjorken-Johnson-Low (BJL) procedure to the correlation function, stating that the $1 / p_{0}$ term in the matrix element of the two-point function, at large $p_{0}$, determines the commutator,

$$
\lim _{p_{0} \rightarrow \infty} p_{0} \int d t e^{i p_{0}\left(t-t_{1}\right)}\left\langle i\left|\hat{l}_{a}(t) \hat{l}_{a_{1}}\left(t_{1}\right)\right| j\right\rangle=i\left\langle i\left|\left[\hat{l}_{a}, \hat{l}_{a_{1}}\right]\left(t_{1}\right)\right| j\right\rangle,
$$

where $\left[\hat{l}_{a}, \hat{l}_{a_{1}}\right]$ is the ETC between field operators corresponding to $l_{a}, l_{a_{1}}$. Integrating the left-hand side in the last expression by parts, we rewrite it as

$$
i \lim _{p_{0} \rightarrow \infty} \int d t e^{i p_{0}\left(t-t_{1}\right)} \frac{\partial}{\partial t}\left\langle i\left|\hat{l}_{a}(t) \hat{l}_{a_{1}}\left(t_{1}\right)\right| j\right\rangle
$$

where we neglected boundary terms. From (30), the last expression is equal to

$$
\begin{aligned}
& -\lim _{p_{0} \rightarrow \infty} \int d t e^{i p_{0}\left(t-t_{1}\right)} f_{a a_{1}}{ }^{b}\left\langle i\left|\hat{l}_{b}(t)\right| j\right\rangle \delta\left(t-t_{1}\right) \\
& =-\lim _{p_{0} \rightarrow \infty} f_{a a_{1}}{ }^{b}\left\langle i\left|\hat{l}_{b}\left(t_{1}\right)\right| j\right\rangle,
\end{aligned}
$$

so that from (31) one gets

$$
\left[\hat{l}_{a}, \hat{l}_{b}\right]=i f_{a b} c \hat{l}_{c}
$$

or, expanding in terms of the operators $\hat{v}, \hat{j}$,

$$
\begin{aligned}
{\left[\hat{v}_{\mu}, \hat{v}_{\nu}\right] } & =i \hat{j}_{\mu \nu}, \quad\left[\hat{j}_{\mu \nu}, \hat{v}_{\rho}\right]=i\left(\hat{v}_{\nu} \eta_{\mu \rho}-\hat{v}_{\mu} \eta_{\nu \rho}\right), \\
{\left[\hat{j}_{\mu \nu}, \hat{j}_{\rho \sigma}\right] } & =i\left(\eta_{\mu \rho} \hat{j}_{\nu \sigma}+\eta_{\nu \sigma} \hat{j}_{\mu \rho}-\eta_{\mu \sigma} \hat{j}_{\nu \rho}-\eta_{\nu \rho} \hat{j}_{\mu \sigma}\right) .
\end{aligned}
$$

Finally, using the substitution (17), we can rewrite the commutators as

$$
\begin{aligned}
{\left[\hat{v}_{\mu}, \hat{v}_{\nu}\right] } & =\hat{S}_{\mu \nu}, \quad\left[\hat{S}_{\mu \nu}, \hat{v}_{\rho}\right]=\hat{v}_{\mu} \eta_{\nu \rho}-\hat{v}_{\nu} \eta_{\mu \rho} \\
{\left[\hat{S}_{\mu \nu}, \hat{S}_{\rho \sigma}\right] } & =\eta_{\mu \sigma} \hat{S}_{\nu \rho}+\eta_{\nu \rho} \hat{S}_{\mu \sigma}-\eta_{\mu \rho} \hat{S}_{\nu \sigma}-\eta_{\nu \sigma} \hat{S}_{\mu \rho} .
\end{aligned}
$$

This is nothing but the Lie algebra (with real structure constants) $\mathfrak{S} \mathfrak{o}(4,1)$ of $S O(4,1)$, which proves that, after computing the path integral in the formula (28), the operators $\hat{v}_{\mu}$ can be taken to be a matrix of a particular representation of the $\mathfrak{s} \mathfrak{o}(4,1)$ algebra. We will show in the next session how, depending on the specific $\mathfrak{g} \mathfrak{o}(4,1)$ representation, one gets in this way the Dirac (spin-1/2) or the DKP (spin-0 or spin-1) propagator (and presumably the propagator for higher spins as well).

To complete the derivation, we need yet another property of the correlation function (28) derived in Appendix C,

$$
\left\langle i\left|\exp \left(i p_{\mu} \int_{0}^{L} \hat{v}^{\mu}(t) d t\right)\right| j\right\rangle=\langle i|\exp (i L p \cdot \hat{v})| j\rangle .
$$

Now, we can integrate (37) over $L$ to find the momentum space propagator,

$$
G_{i j}(p)=\left\langle i\left|\frac{i}{p \cdot \hat{v}-m-i \epsilon}\right| j\right\rangle .
$$

\section{THE PROPAGATOR FOR DIFFERENT SPINS}

Depending on the specific choice of representation for the $\mathfrak{S} \mathfrak{v}(4,1)$ generators, expression (38) gives the propagator for different spin values in the first order formalism. As shown in Appendix B, the spinning term $\exp \left(i \int \omega\right)$, upon appropriate choice of coadjoint orbits, decomposes the path integral into matrix elements between states belonging to the finite dimensional representations of $\mathrm{SO}(4,1)$ labeled by the highest weights of the irreducible representations of the maximally compact subgroup $\mathrm{SO}(4) \simeq \mathrm{SU}(2) \otimes \mathrm{SU}(2)$, parametrized by a set of ordered integer or half-integer numbers, ${ }^{5}$

\footnotetext{
${ }^{5}$ The characterization of these finite dimensional representations is carried out in Appendix B. The classification of irreducible unitary (infinite dimensional) representations of $\mathrm{SO}(4,1)$ induced from the maximal compact subgroup was accomplished in [17] following the method developed in $[18,19]$, exploiting the relation between representations of the group and of its Lie algebra. The analogous characterization for the Euclidean case, leading to (finite dimensional) irreps of SO (5), is carried out in [20].
} 


$$
(p, q): p \geq q \geq 0
$$

Following the argument worked out in [21] for the Euclidean case, we can define the algebras $\mathcal{B}^{(k)}$ arising from the $\mathfrak{s} \mathfrak{v}(4,1)$ matrix representations $\pi_{p, q}\left(\hat{v}_{\mu}\right)$ defined in Appendix B, satisfying the commutation relations, following from (36),

$$
\left[\left[\hat{v}_{\mu}, \hat{v}_{\nu}\right], \hat{v}_{\rho}\right]=\hat{v}_{\mu} \eta_{\nu \rho}-\hat{v}_{\nu} \eta_{\mu \rho} .
$$

and, for $k \geq p$, the equation [following from (B11)]

$$
\prod_{m=-k}^{k}\left(\hat{v}_{\mu}-\left(\delta_{\mu 0}+i \sum_{i} \delta_{\mu i}\right) m \mathbb{1}\right)=0 .
$$

Different values of $k$ then correspond to different spin sectors.

For $k=1 / 2$, one has a four-dimensional $\pi\left(\frac{1}{2}, \frac{1}{2}\right)$ representation (see Appendix B) of $\mathcal{B}^{\left(\frac{1}{2}\right)}$ corresponding to the Dirac algebra. Indeed, Eq. (41) becomes $\hat{v}_{\mu}^{2}=-\frac{1}{4} \eta_{\mu \mu}$, and defining $\gamma_{\mu}=2 \hat{v}_{\mu}$, we find from (40) that

$$
\gamma_{\mu} \gamma_{\nu}+\gamma_{\nu} \gamma_{\mu}=2 \eta_{\mu \nu}
$$

Plugging this to (38), we get the spin- $\frac{1}{2}$ propagator,

$$
G(p) \propto \frac{1}{p_{\mu} \gamma^{\mu}-m}\left(=\frac{p_{\mu} \gamma^{\mu}+m}{p^{2}-m^{2}}\right) .
$$

It appears that the spin- $-\frac{1}{2}$ propagator has its usual form expressed in terms of $p$ momenta.

For $k=1$, the matrices $\beta_{\mu}$ (no summation) satisfy the relations that define the DKP algebra [11-13],

$$
\beta_{\mu} \beta_{\rho} \beta_{\nu}+\beta_{\nu} \beta_{\rho} \beta_{\mu}=\beta_{\mu} \eta_{\nu \rho}+\beta_{\nu} \eta_{\mu \rho} .
$$

The derivation of Eq. (44) is carried out in Appendix D. In this case, one has three irreducible representations (see Appendix B), the trivial one-dimensional $\pi(0,0)$, the fivedimensional $\pi(1,0)$, and the ten-dimensional $\pi(1,1)$. Several results (see, for instance, [14] and [22]) have been obtained showing that for these two latter irreducible representations the DKP field equations reduce, respectively, to the equations of motion for a spin- 0 scalar field (the Klein-Gordon equation) and for a spin-1 vector field (the Proca equations). However, to our knowledge, the reduction of the propagator to the standard expressions for the spin-0 and spin- 1 fields have not been treated thoroughly, and we devote next section to this task.

\section{A. The propagator for spin- 0 and spin-1}

Let us start noticing that it follows from (38) that the propagator in momentum space is (apart from the term $i \epsilon$ ) the inverse of the matrix $\not p-m$, where we denote $\not p=p_{\mu} \beta^{\mu}$, i.e.,

$$
G(p)=(\not p-m \mathbb{1})^{-1} .
$$

Using the properties (44) of the DKP matrices, one can prove that (see, for instance, [23])

$$
G(p)=\frac{1}{m}\left(\frac{\not p(\not p+m)}{p^{2}-m^{2}}-\mathbb{1}\right) .
$$

Indeed, from (44),

$$
\not p^{3}=p_{\mu} p_{\rho} p_{\nu} \beta^{\mu} \beta^{\rho} \beta^{\nu}=p_{\mu} p_{\rho} p_{\nu} \beta^{\mu} \eta^{\rho \nu}=\not p p^{2} .
$$

Then,

$\not \not(\not p+m \mathbb{1})(\not p-m \mathbb{1})=\left(\not \not^{3}-m^{2} \not \not\right)=\not p\left(p^{2}-m^{2}\right)$,

and it follows

$$
G(p)(\not p-m \mathbb{1})=\frac{1}{m}(\not p-(\not p-m \mathbb{1}))=\mathbb{1} .
$$

We consider first the five-dimensional representation $\pi(1,0)$ describing the spin- 0 sector. The field equations for spin- 0 are obtained with the help of a projection operator [14],

$$
\mathcal{P}=-\beta_{0}^{2} \beta_{1}^{2} \beta_{2}^{2} \beta_{3}^{2},
$$

so that the field

$$
\psi=\left(\begin{array}{l}
\psi_{0} \\
\psi_{1} \\
\psi_{2} \\
\psi_{3} \\
\psi_{4}
\end{array}\right)
$$

decomposes into the vector field $\mathcal{V}^{\mu}=\mathcal{P} \beta^{\mu} \psi$ and the scalar one $\Phi=\mathcal{P} \psi$. Indeed, one can show that $\Phi$ and $\mathcal{V}^{\mu}$ transform, respectively, as a (pseudo)-scalar and a (pseudo)-vector under Lorentz transformations, where infinitesimal transformations are generators by $S_{\mu \nu}=$ $\left[\beta_{\mu}, \beta_{\nu}\right]$ as $\left(\omega^{\mu \nu}=-\omega^{\mu \nu}\right)$,

$$
U \simeq 1+\frac{1}{2} \omega^{\mu \nu} S_{\mu \nu}
$$

Moreover, one can show that upon imposing the DKP equation for the free field $\psi$, the components of $\psi$ are not independent, and that one can define (see Appendix D) a specific representation of the $\beta^{\mu}$ such that $\psi_{4}=\phi$ and $\psi_{\mu}=\partial_{\mu} \phi$, making explicit the fact that $\psi$ describes in this case the scalar $\phi$ and its derivatives $\partial_{\mu} \phi$.

We can obtain the propagator for the scalar field $\mathcal{S}(p)$ by projecting the propagator (46) on the scalar field sector with $\mathcal{P}$, 


$$
\mathcal{S}(p) \equiv \frac{1}{m} \mathcal{P} G(p) \mathcal{P}^{\dagger},
$$

so that $\mathcal{S}(p)$ is defined by the matrix element in (the mass factor is for dimensional reasons)

$$
\bar{\Phi} \mathcal{P} G(p) \mathcal{P}^{\dagger} \Phi=m \bar{\Phi} \mathcal{S}(p) \Phi
$$

As discussed in Appendix B, since we are in Lorentzian metric, the $\beta_{0}$ and $\beta_{j}$ matrices must have opposite hermiticity, and in particular in our notations we have that $\beta_{0}$ is Hermitian and $\beta_{j}$ anti-Hermitian: $\beta_{0}^{\dagger}=\beta_{0}, \beta_{j}^{\dagger}=-\beta_{j}$.

In DKP theory, the adjoint field is given by $\bar{\psi}=\psi^{\dagger} \eta_{0}$, where $\eta_{\mu}$ is the operator,

$$
\eta_{\mu}=2 \beta_{\mu}^{2}-\eta_{\mu \mu}
$$

such that $\beta_{\mu}^{\dagger}=\eta_{0} \beta_{\mu} \eta_{0}$ and $\bar{\Phi}=\psi^{\dagger} \eta_{0} \mathcal{P}^{\dagger}$. Noticing also that from the defining properties of the $\beta$ matrices (44), setting $\mu=\nu$ in (44), follow the relations

$$
\beta_{\mu} \beta_{\nu} \beta_{\mu}=\beta_{\mu} \eta_{\mu \nu}
$$

one finds that

$$
\mathcal{P} \not \mathcal{P}^{\dagger}=p^{\mu} \mathcal{P} \beta_{\mu} \mathcal{P}^{\dagger}=0 .
$$

Using again (44) [setting $\nu=\rho \neq \mu$ in (44)], we find the relations

$$
\beta_{\mu} \beta_{\nu}^{2}+\beta_{\nu}^{2} \beta_{\mu}=\beta_{\mu} \eta_{\nu \nu} \quad \mu \neq \nu,
$$

and multiplying last relation by $\beta_{\mu}$ from the left and from the right, we find

$$
\beta_{\mu}^{2} \beta_{\nu}^{2}=\beta_{\nu}^{2} \beta_{\mu}^{2}
$$

From last relation, the hermiticity of $\beta$ 's and (56), we also find that

$$
\mathcal{P}^{\dagger}=\mathcal{P}, \quad \mathcal{P}^{2}=\mathcal{P},
$$

while using (59) and (56), it follows

$$
\mathcal{P} \beta^{\mu} \beta^{\nu}=\mathcal{P} \eta^{\mu \nu}
$$

From last relations, we find

$$
\mathcal{P} \not p \not \mathcal{P}^{\dagger}=p^{2} \mathcal{P} \mathcal{P}^{\dagger}=p^{2} .
$$

Plugging (57) and (62) together with (46) in (54), we finally obtain

$$
\begin{aligned}
\frac{1}{m} \bar{\Phi} \mathcal{P} G(p) \mathcal{P}^{\dagger} \Phi & =\frac{1}{m^{2}} \bar{\Phi} \mathcal{P}\left(\frac{\not p(\not p+m)}{p^{2}-m^{2}}-\mathbb{1}\right) \mathcal{P}^{\dagger} \Phi \\
& =\bar{\Phi} \frac{1}{p^{2}-m^{2}} \Phi
\end{aligned}
$$

so that

$$
\mathcal{S}(p)=\frac{1}{p^{2}-m^{2}}
$$

The DKP propagator, projected on the scalar field sector, has the standard form.

We can repeat a similar procedure to derive the propagator for the spin-1 representation. In this case, the projection operators are

$$
\mathcal{R}_{\mu}=\beta_{1}^{2} \beta_{2}^{2} \beta_{3}^{2}\left(\beta_{\mu} \beta_{0}-\eta_{\mu 0}\right),
$$

where now the $\beta$ matrices are to be taken in the tendimensional irreducible representation (we give an explicit realization in Appendix D). The beta matrices maintain the same hermiticity of the scalar case, and one can show that $\mathcal{R}_{\mu} \psi$ transforms, under the infinitesimal Lorentz transformation (52), like a (pseudo)vector while $\mathcal{R}_{\mu \nu} \psi=$ $\mathcal{R}_{\mu} \beta_{\nu} \psi$ like a (pseudo)tensor. Upon imposing the DKP equation for $\psi$, one can then show that $\mathcal{R}_{\mu \nu}$ is proportional to the strength tensor of the vector field $R_{\mu} \psi$ (see, for instance, [14] and [22]). We define then the vector field $\mathcal{A}_{\mu}$ and its adjoint as

$$
\mathcal{A}_{\mu}=\mathcal{R}_{\mu} \psi, \quad \overline{\mathcal{A}}_{\mu}=-\bar{\psi} \mathcal{R}_{\mu}^{\dagger}=-\psi^{\dagger} \eta_{0} \mathcal{R}_{\mu}^{\dagger},
$$

with $\eta_{0}$ given by (55). It is possible to show that with this definition the fields $\mathcal{A}_{\mu}$ and $\overline{\mathcal{A}}_{\mu}$ transform, respectively, with covariant and contravariant indexes. Thus, we may identify

$$
\mathcal{A}^{\mu}=\overline{\mathcal{A}}_{\mu}
$$

( $\sum_{\mu} \overline{\mathcal{A}}_{\mu} \mathcal{A}_{\mu}=\mathcal{A}_{\mu} \mathcal{A}^{\mu}$ transforms as a (pseudo)scalar). The spin-1 propagator $\mathcal{S}^{\mu \nu}(p)$ for the vector field is then obtained by projection

$$
\mathcal{S}_{\mu}{ }^{\nu}(p) \equiv \frac{1}{m} \mathcal{R}_{\mu} G(p) \mathcal{R}_{\nu}^{\dagger}
$$

as the matrix element in

$$
\sum_{\mu, \nu} \overline{\mathcal{A}}_{\mu} \mathcal{R}_{\mu} G(p) \mathcal{R}_{\nu}^{\dagger} \mathcal{A}_{\nu}=m \mathcal{A}_{\mu} \mathcal{S}^{\mu \nu}(p) \mathcal{A}_{\nu} .
$$

We can use the properties of the $\beta$ matrices (56), (58), and (59) to find the following relations: 


$$
\begin{aligned}
\mathcal{R}_{\mu} \mathcal{R}_{\nu}^{\dagger} & =\delta_{\mu}^{\nu} \mathcal{R}_{0}, \quad \mathcal{R}_{0} \mathcal{R}_{\mu}=\mathcal{R}_{\mu} \quad \mathcal{R}_{\mu} \beta_{\rho} \mathcal{R}_{\nu}^{\dagger}=0, \\
\mathcal{R}_{\mu} \beta_{\rho} \beta_{\sigma} & =\eta_{\rho \sigma} \mathcal{R}_{\mu}-\eta_{\mu \sigma} \mathcal{R}_{\rho} .
\end{aligned}
$$

Using these relations, it follows that

$$
\begin{aligned}
\mathcal{R}_{\mu} \not p \mathcal{R}_{\nu}^{\dagger} & =0, \\
\mathcal{R}_{\mu} \not \not p \mathcal{R}_{\nu}^{\dagger} & =p^{2} \mathcal{R}_{\mu} \mathcal{R}_{\nu}^{\dagger}-p_{\mu} p^{\rho} \mathcal{R}_{\rho} \mathcal{R}_{\nu}^{\dagger}
\end{aligned}
$$

and finally

$$
\begin{aligned}
& \frac{1}{m} \overline{\mathcal{A}}_{\mu} \mathcal{R}_{\mu} G(p) \mathcal{R}_{\nu}^{\dagger} \mathcal{A}_{\nu} \\
& =\frac{1}{m^{2}} \overline{\mathcal{A}}_{\mu} \mathcal{R}_{\mu}\left(\frac{\not p(\not p+m)}{p^{2}-m^{2}}-\mathbb{1}\right) \mathcal{R}_{\nu}^{\dagger} \mathcal{A}_{\nu} \\
& =\frac{1}{m^{2}\left(p^{2}-m^{2}\right)} \overline{\mathcal{A}}_{\mu}\left(m^{2} \mathcal{R}_{\mu} \mathcal{R}_{\nu}^{\dagger}-p_{\mu} p^{\rho} \mathcal{R}_{\rho} \mathcal{R}_{\nu}^{\dagger}\right) \mathcal{A}_{\nu} \\
& =\frac{1}{m^{2}\left(p^{2}-m^{2}\right)} \overline{\mathcal{A}}_{\mu}\left(m^{2} \delta_{\mu}{ }^{\nu}-p_{\mu} p^{\nu}\right) \mathcal{R}_{0} \mathcal{A}_{\nu} \\
& =\mathcal{A}_{\mu} \frac{1}{p^{2}-m^{2}}\left(\eta^{\mu \nu}-\frac{p^{\mu} p^{\nu}}{m^{2}}\right) \mathcal{A}_{\nu},
\end{aligned}
$$

where we used the above relations together with $\mathcal{R}_{0} \mathcal{A}_{\mu}=$ $\mathcal{R}_{0} \mathcal{R}_{\mu} \psi=\mathcal{R}_{\mu} \psi=\mathcal{A}_{\mu}$. Thus, the spin-1 propagator reduces to the standard propagator in unitary gauge,

$$
\mathcal{S}^{\mu \nu}(p)=\eta^{\mu \nu}-\frac{p^{\mu} p^{\nu}}{m^{2}}
$$

\section{CONCLUSIONS}

In this paper, following the idea of Alekseev and Shatashvili of adding to the first order action the Kirillov presymplectic form, which forces the path integral to select a particular representation of the de Sitter group, we derive the DKP propagator for fields of spin- 0 and spin-1 (as well as the Dirac propagator for spin-1/2) in the path integral formalism. We then show that the obtained DKP propagators are equivalent to the standard ones.

There are several interesting problems that could be addressed in follow-up investigations. First, although it seems pretty obvious that an analogous construction should work for spins higher than 1, it would be illuminating to do it explicitly.

Second, the construction presented here can, presumably, be extended to the case of $\kappa$-deformation (in the sense of $\kappa$-Poincaré Hopf symmetries) [24-27], a scenario that has attracted much interest especially in relation with quantum gravity phenomenology. In this case, momentum space is not the ordinary flat (Minkowskian) momentum space, but it is described as a curved manifold (specifically the group $\mathrm{AN}_{3}$, corresponding to half of de Sitter space, see [27]), whose scale of curvature $1 / \kappa$ is taken to be proportional to the (inverse) Planck energy $\left(1 / E_{\mathrm{pl}} \sim 10^{-19} \mathrm{GeV}\right)$.
As (four-dimensional) $\kappa$-momentum space can be also described in terms of flat embedding "momentum coordinates" in five dimensions, with some additional constraint enforcing physical momenta to live on the de Sitter hyperboloid, one can think of extending the formalism described in this paper, which is not restricted to fourdimensional momentum space, exploiting the use of embedding coordinates. The construction of a Dirac (spin-1/2) action with $\kappa$-Poincaré symmetries has been already addressed in some previous works (see, for instance, $[25,28-30])$. It would be interesting to compare the spin-1/2 propagator for $\kappa$-momentum space resulting from our approach with previous results. Moreover, if working, our construction would allow in principle to study higher-spin propagators for $\kappa$-momentum space, setting the stage for constructing a higher-spin field theory action based on $\kappa$-deformed symmetries.

\section{ACKNOWLEDGMENTS}

This work is supported by the Polish National Science Centre Grant No. 2019/33/B/ST2/00050 and for J. K. G. also by the Polish National Science Centre Grant No. 2017/ 27/B/ST2/01902.

\section{APPENDIX A: THE ACTION FUNCTIONAL ON THE ORBITS}

We here discuss the construction of the action (24) needed to implement the spin degrees of freedom in the path integral. We refer the reader to the characterization outlined, for instance, in [31], based on the Kirillov symplectic form [32]. Consider a (matrix) Lie group $G$. Let $\mathfrak{g}$ be Lie algebra of $G$ and $\mathfrak{g}^{*}$ its dual Lie algebra: for a basis $\left\{e_{a}\right\}$ of $\mathfrak{g}$ and $\left\{\tilde{e}^{a}\right\}$ of $\mathfrak{g}^{*}$, the duality relations are canonically given by $\left\langle\tilde{e}^{a}, e_{b}\right\rangle=\delta_{b}^{a}$. The coadjoint representation of $G$ is defined by

$$
\begin{aligned}
\left\langle\operatorname{Ad}^{*}(g) X, u\right\rangle & =\left\langle X, \operatorname{Ad}(g)^{-1} u\right\rangle, \quad \text { where } \operatorname{Ad}(g) u=g u g^{-1}, \\
\left\langle\operatorname{ad}^{*}(u) X, v\right\rangle & =-\langle X, \operatorname{ad}(u) v\rangle, \quad \text { where } \operatorname{ad}(u) b=[u, v],
\end{aligned}
$$

where $g \in G, X \in \mathfrak{g}^{*}, u, v \in \mathfrak{g}$. Let us parametrize the orbits by group variables fixing the point $X_{0}$ so that a generic point on the orbit is

$$
X(g)=\operatorname{Ad}^{*}(g) X_{0} .
$$

In the basis $\left\{e_{a}\right\}$ and $\left\{\tilde{e}^{a}\right\}$, we will write a generic point on the orbit as $X=l_{a} \tilde{e}^{a}$. Define the action

$$
S=\int \omega, \quad \omega=\langle X(g), Y(g)\rangle,
$$

with

$$
Y(g)=d g g^{-1} \in \mathfrak{g}
$$


Here $Y=d g g^{-1}$ is the Maurer-Cartan form on the group. It is possible to show that the following equivalent equations are satisfied:

$$
\begin{aligned}
& d X=\operatorname{ad}^{*}(Y) X, \\
& d l_{a}=f_{a b}{ }^{c} Y^{b} l_{c},
\end{aligned}
$$

where $X=l_{a} \tilde{e}^{a}$, and $Y=Y^{a} e_{a}$, and $f_{a b}{ }^{c}$ are the structure constant of the Lie algebra ${ }^{6} \mathfrak{g}\left[e_{a}, e_{b}\right]=f_{a b}{ }^{c} e_{c}$. Let us first show the equivalence of Eqs. (A6) and (A5),

$$
\begin{aligned}
d l_{a} & =\left\langle d X, e_{a}\right\rangle=\left\langle\operatorname{ad}^{*}(Y) X, e_{a}\right\rangle=\left\langle X,\left[e_{a}, Y\right]\right\rangle \\
& =l_{c} Y^{b}\left\langle\tilde{e}^{c},\left[e_{a}, e_{b}\right]\right\rangle=l_{c} Y^{b} f_{a b}{ }^{d}\left\langle\tilde{e}^{c}, e_{d}\right\rangle=f_{a b}{ }^{c} Y^{b} l_{c} .
\end{aligned}
$$

Let us now prove Eq. (A5),

$$
\begin{aligned}
\left\langle d X, e_{a}\right\rangle & =d\left\langle\operatorname{Ad}^{*}(g) X_{0}, e_{a}\right\rangle=\left\langle X_{0}, g^{-1} e_{a} d g+d g^{-1} e_{a} g\right\rangle \\
& =\left\langle\operatorname{Ad}^{*}(g) X_{0},\left[e_{a}, d g g^{-1}\right]\right\rangle=-\left\langle X, \operatorname{ad}(Y) e_{a}\right\rangle \\
& =\left\langle\operatorname{ad}^{*}(Y) X, e_{a}\right\rangle,
\end{aligned}
$$

where we used definition (A2) and $d \mathbb{1}=d\left(g^{-1} g\right)=$ $d g^{-1} g+g^{-1} d g=0$, from which follows $d g^{-1}=-g^{-1} d g g^{-1}$.

Equation (A5) [or (A6)] ensures that the action (A3) generates on the orbit the canonical two-form,

$$
\Omega=-d \omega=\langle X, Y \wedge Y\rangle,
$$

where $d \Omega$ is closed on the orbit. The last equation can be rewritten explicitly as

$$
\begin{aligned}
\Omega & =-d \omega=\left\langle X_{0}, g^{-1} d g \wedge g^{-1} d g\right\rangle \\
& =\left\langle X, d g g^{-1} \wedge d g g^{-1}\right\rangle,
\end{aligned}
$$

where the Maurer-Cartan equation $d\left(g d g^{-1}\right)=-g d g^{-1} \wedge$ $g d g^{-1}$ has been used. One can show that the Poisson brackets of the restriction of the linear functions on the orbit reproduce the algebraic commutation relation. Defining the linear functions $u(X)=\langle X, u\rangle$, where on the r.h.s. $u=$ $u^{a} e_{a}$ (so that $u(X)=u^{a} l_{a}$ ), we get

$$
\begin{aligned}
\{u(X), v(X)\} & =\Omega(u, v)=\langle X,[Y(u), Y(v)]\rangle \\
& =u^{a} v^{b}\left\langle X,\left[e_{a}, e_{b}\right]\right\rangle=f_{a b}{ }^{c} u^{a} v^{b} l_{c},
\end{aligned}
$$

where we used that the Maurer-Cartan form evaluated on an element of the basis of the Lie algebra gives $Y\left(e_{a}\right)=e_{a}$. It follows in particular, for $u^{a}=\delta_{b}^{a}$, that on the orbit,

$$
\left\{l_{a}, l_{b}\right\}=f_{a b}^{c} l_{c} .
$$

\footnotetext{
${ }^{6}$ Here, $[\cdot, \cdot]$ denotes obviously the Lie bracket.
}

\section{APPENDIX B: COADJUST ORBITS AND IRREPS FOR SO $(4,1)$}

\section{Integral orbits for $\mathrm{SO}(3,2)$}

In the spirit of geometric quantization (see, for instance, $[33,34])$, the orbit method [32] can be used to quantize the values of some parameters labeling the orbits of the action of the group on its dual Lie algebra. This mechanism can be realized $[7,16]$ by the requirement for the action exponential $\exp (i S(\ell))$ to be single valued, so that the path integral is well defined. Indeed, the one-form $\omega$ is singular, and the action $S=\int \omega$ is multivalued, and the requirement of uniqueness of the expression $\exp (i S(\ell))$ over closed path leads to integral orbits.

In our specific case, starting from the $\mathfrak{g} \mathfrak{v}(3,2)$ algebra (20), so that a generic element can be parametrized as $u=\tilde{v}^{\mu} \Upsilon_{\mu}+\frac{1}{2} \tilde{j}^{\mu \nu} J_{\mu \nu}\left(\tilde{j}^{\nu \mu}=-\tilde{j}^{\mu \nu}\right)$, we can fix the orbits considering the action of the Lorentz subgroup $\mathrm{SO}(3,1)$ generated by $J_{\mu \nu}$. Rewriting the generators as $R_{i}=$ $-\frac{1}{2} \epsilon_{i j k} J_{j k}$ and $P_{i}=\Upsilon_{i}$, we can rewrite the $\mathfrak{g} \mathfrak{o}(3,1)$ subalgebra as

$\left[R_{i}, R_{j}\right]=\epsilon_{i j k} R_{k}, \quad\left[R_{i}, P_{j}\right]=\epsilon_{i j k} P_{k}, \quad\left[P_{i}, P_{j}\right]=-\epsilon_{i j k} R_{k}$,

so that an element of the $\mathfrak{g} \mathfrak{o}(3,1)$ subalgebra is $u_{\mathfrak{S} \mathfrak{o}(3,1)}=$ $r^{i} R_{i}+p^{i} P_{i}$, with $r^{i}=-\epsilon_{j k}^{i} \tilde{j}^{j k}, p^{i}=\tilde{v}^{i}$. Reparametrizing (see [15], Ch. 5.6) an element of $\mathfrak{g} \mathfrak{o}(3,1)$ as $u_{\mathfrak{g} \mathfrak{o}(3,1)}=$ $a^{i} A_{i}+b^{i} B_{i}$, with $A_{i}=\frac{1}{2}\left(R_{i}+i P_{i}\right), B_{i}=\frac{1}{2}\left(R_{i}-i P_{i}\right)$, so that $a^{i}=r^{i}+i p^{i}, b^{i}=r^{i}-i p^{i}$, the algebra splits into a direct sum of two mutually commuting complex (conjugate) $\quad \mathfrak{g} \mathfrak{t}(2): \quad \mathfrak{g} \mathfrak{v}(3,1) \approx \mathfrak{g} \mathfrak{t}(2)_{\mathbb{C}} \oplus \overline{\mathfrak{g} \mathfrak{t}(2)} \mathbb{C} \approx \mathfrak{g l}(2, \mathbb{C}) \oplus$ $\overline{\mathfrak{G l}(2, \mathbb{C})}$. Thus, we have reduced the problem to fixing the orbits of the two $\operatorname{Sl}(2, \mathbb{C})$ subgroups of $S O(3,1)$. Finally, we notice that each of the two $\operatorname{Sl}(2, \mathbb{C})$ admits $S U(2)$ as (maximal) compact subgroup, and we can use it to fix the orbits for each of the two copies.

Representing the $\mathrm{SU}(2)$ generators in terms of Pauli matrices, $A_{i}, B_{i} \equiv-\frac{i}{2} \sigma_{i}$,

$\sigma_{1}=\left(\begin{array}{cc}0 & 1 \\ 1 & 0\end{array}\right), \quad \sigma_{2}=\left(\begin{array}{cc}0 & -i \\ i & 0\end{array}\right), \quad \sigma_{3}=\left(\begin{array}{cc}1 & 0 \\ 0 & -1\end{array}\right)$,

for each $\mathrm{SU}(2)$ copy we can parametrize an element of the group by Euler angles as

$g_{\mathrm{SU}(2)}=\exp \left(-\frac{i}{2} \sigma_{3} \chi\right) \exp \left(-\frac{i}{2} \sigma_{2} \theta\right) \exp \left(-\frac{i}{2} \sigma_{3} \phi\right)$,

with 


$$
\chi \in[0,2 \pi), \quad \theta \in[0, \pi], \quad \phi \in[0,2 \pi) .
$$

On each copy, the Maurer-Cartan connection $Y_{\mathrm{SU}(2)}=$ $g_{\mathrm{SU}(2)}^{-1} d g_{\mathrm{SU}(2)}$ can be evaluated to

$$
Y_{\mathrm{SU}(2)}^{i}=\left(\begin{array}{c}
-\sin \chi d \theta+\cos \phi \sin \theta d \phi \\
\cos \chi d \theta+\sin \phi \sin \theta d \phi \\
d \chi+\cos \theta d \phi
\end{array}\right),
$$

where $Y=Y^{i} A_{i}$ or $Y=Y^{i} B_{i}$. The orbits can be chosen fixing the value of the coordinates in $\mathfrak{g}_{\mathfrak{a} \mathfrak{u}(2)}^{*} \tilde{a}=\tilde{a}_{i} \tilde{A}^{i}$, $\tilde{b}=\tilde{b}_{i} \tilde{A}^{i}$ along the (real) $z$ direction $\left(\left\langle\tilde{A}^{i}, A_{j}\right\rangle=\delta_{j}^{i}\right.$, $\left.\left\langle\tilde{B}^{i}, B_{j}\right\rangle=\delta_{j}^{i}\right), \quad \operatorname{Re}\left(\tilde{a}_{i}\right)=(0,0, m), \quad \operatorname{Re}\left(\tilde{b}_{i}\right)=(0,0, n)$, and we thus find, respectively, the action $(s=m$ or $n)$,

$$
\int \omega_{S U(2)}=\gamma \int d \phi+s \int \cos \theta d \phi
$$

where we renamed the azimuthal angle $\chi=\gamma^{\prime} \phi$ for some constant $\gamma^{\prime}$ and $\gamma=\gamma^{\prime} s$. The action is multivalued as it counts the windings around the axis passing through the poles $\theta=0, \pi$ of the sphere, where the one-form $\cos \theta d \phi$ is singular. For infinitesimal closed contours around the poles $\theta=0, \pi$, the action gives the value $2 \pi(\gamma \pm s)$, so that, if $\gamma \pm s$ is an integer, the action $\exp \left(i \int \omega\right)$ does not contribute to the path integral, which is then well defined. We can choose $\gamma=0$ for $s$ integer and $\gamma=\frac{1}{2}$ for $s$ semi-integer. Thus, the condition for single valuedness of $\exp \left(i \int \omega\right)$ translates into the condition of "quantization" of the values of $s=(m, n)$, which take only integer or semi-integer values $\left(\ell_{1}, \ell_{2}\right)$.

\section{Discrete series and finite dimensional representations of $\operatorname{SO}(4,1)$}

With this choice of orbits, after quantization [see Eq. (35)], the elements $l_{a} \equiv\left(v_{\mu}, j_{\mu \nu}\right) \rightarrow\left(\hat{v}_{\mu}, \hat{j}_{\mu \nu}\right)$ of the dual algebra belong to one of the irreducible unitary representations of $\mathfrak{s} \mathfrak{v}(3,2)$ induced by the (real structure) decomposition $\mathfrak{g} \mathfrak{u}(2) \oplus \overline{\mathfrak{S} \mathfrak{t}(2)}$. Finally, after the substitution (17) $\left(\hat{S}_{\mu \nu}=i \hat{j}_{\mu \nu}\right)$, the (matrix) operators $\hat{v}_{\mu}$ and $S_{\mu \nu}$ belong to one of the irreducible unitary representations of $\mathfrak{g} \mathfrak{\mathfrak { v }}(4,1)$, seen as a Lie algebra with real structure constants, Eq. (36), induced by the maximal compact subgroup $\mathrm{SO}(4) \approx \mathrm{SU}(2) \oplus \mathrm{SU}(2)$. The classification of such representations has been carried out in [17] perfecting an approach developed previously in $[18,19]$ relating the group representation to the representation of its Lie algebra generators. In particular, it is shown that the discrete series representation $\pi_{\tilde{p}, \tilde{q}}$ of $\mathrm{SO}(4,1)$ can be obtained in this way (see also [35]). The discrete series $\pi_{\tilde{p}, \tilde{q}}$ is labeled by two integers or semi-integers values $(\tilde{p}, \tilde{q})$ related to the $\mathrm{SO}(4) \simeq \mathrm{SU}(2) \otimes \mathrm{SU}(2) \quad$ labels by $\tilde{p}=\inf _{\left(\ell_{1}, \ell_{2}\right) \in \Gamma} \times$ $\left(\ell_{1}+\ell_{2}\right), \tilde{q}=\inf _{\left(\ell_{1}, \ell_{2}\right) \in \Gamma}\left(\ell_{1}-\ell_{2}\right)$, where $\Gamma$ is the set of values $\left(\ell_{1}, \ell_{2}\right)$ which occur in the reduction $\left.\pi_{\tilde{p}, \tilde{q}}\right|_{S O(4)}$ of the representation to $\mathrm{SO}(4)$, and $\tilde{p}=\frac{1}{2}, 1, \frac{3}{2}, 2, \ldots$, $\tilde{q}=\tilde{p}, \tilde{p}-1, \ldots, 1$ or $\frac{1}{2}$. The Hilbert space of the representation is the infinite ${ }^{7}$ direct sum $\mathcal{H}=\oplus_{\left(\ell_{1}, \ell_{2}\right) \in \Gamma}$ $\mathcal{H}_{\ell_{1}, \ell_{2}}$ of the subspaces $\mathcal{H}_{\ell_{1}, \ell_{2}}$ invariant under $\pi_{\tilde{p}, \tilde{q}}(\mathrm{SO}(4))$. Each representation $\pi_{\tilde{p}, \tilde{q}}$ is characterized by a different value of the two Casimir invariants of the $\mathfrak{g} \mathfrak{o}(4,1)$ Lie algebra corresponding to the ones in $(22)$ of $\mathfrak{s} \mathfrak{p}(3,2)$,

$$
\begin{aligned}
& \hat{\mathcal{C}}_{1}=\hat{v}^{\mu} \hat{v}_{\mu}-\frac{1}{2} \hat{S}_{\mu \nu} \hat{S}^{\mu \nu}, \\
& \hat{\mathcal{C}}_{2}=\hat{W}_{0}^{2}-\hat{W} \cdot \hat{W}-\frac{1}{4}\left(\epsilon_{i j k} \hat{S}_{j k} \hat{S}_{i 0}\right)^{2},
\end{aligned}
$$

where $\quad \hat{W}_{0}=\frac{1}{2} \epsilon_{i j k} \hat{S}_{j k} \hat{v}_{i}, \quad \hat{W}=-\frac{1}{2} \epsilon_{i j k} \hat{S}_{j k} \hat{v}_{0}+\epsilon_{i j k} \hat{S}_{j 0} \hat{0}_{k}$. Indeed, for a given $\pi_{\tilde{p}, \tilde{q}}, \hat{\mathcal{C}}_{1}$ and $\hat{\mathcal{C}}_{2}$ are scalar operators taking the values [17]

$$
\begin{aligned}
& \pi_{\tilde{p}, \tilde{q}}\left(\hat{\mathcal{C}}_{1}\right)=(\tilde{p})(\tilde{p}+1)-2+(\tilde{q})(\tilde{q}-1), \\
& \pi_{\tilde{p}, \tilde{q}}\left(\hat{\mathcal{C}}_{2}\right)=(\tilde{p})(\tilde{p}+1)(\tilde{q})(\tilde{q}-1) .
\end{aligned}
$$

Thus, the orbit quantization method exhibited in the previous section, singling out integer or semi-integer values $\left(\ell_{1}, \ell_{2}\right)$, selects the values of the invariant polynomials $\mathcal{C}_{1}$ and $\mathcal{C}_{2}$ of $l_{a} \equiv\left(v_{\mu}, j_{\mu \nu}\right)$, defining the orbits, which occur in the irreducible representations of $\mathrm{SO}(4,1)$, and in particular in the discrete series.

Apart from the irreducible unitary (infinite dimensional) representations, one can obtain finite dimensional, nonunitary representations that can be understood as a "Wick rotation" of the irreducible unitary representations of $\mathrm{SO}(5)$ discussed, for instance, in [20]. In this case, the generators can be represented through finite dimensional (mixed Hermitian and anti-Hermitian) matrices, and we will use these representations to construct the projection operators for the different spin sectors of the propagator. Denoting such finite dimensional representations with $\pi_{p, q}$, they are labeled now by $p=\max \left(\ell_{1}+\ell_{2}\right)$ and $q=\max \left(\ell_{1}-\ell_{2}\right)$, whose ranges are such that $p$ and $q$ are all integers or all semi-integers and $p \geq q \geq 0$, while, for given $(p, q), \ell_{1}+$ $\ell_{2}$ and $\ell_{1}-\ell_{2}$ range, respectively, from $q$ to $p$ and from $-q$ to $q$ by steps of 1 (thus, $\ell_{1}, \ell_{2}$ range from 0 to $\frac{1}{2}(p+q)$ by steps of $\frac{1}{2}$ ). The $\pi_{p, q}$ are characterized by the values of the Casimir operators,

$$
\begin{aligned}
& \pi_{p, q}\left(\hat{\mathcal{C}}_{1}\right)=p(p+3)+q(q+1), \\
& \pi_{p, q}\left(\hat{\mathcal{C}}_{2}\right)=(p+1)(p+2) q(q+1),
\end{aligned}
$$

and their dimension is given by the formula

\footnotetext{
${ }^{7}$ Since $\mathrm{SO}(4,1)$ is noncompact, irreducible unitary representations are infinite dimensional.
} 

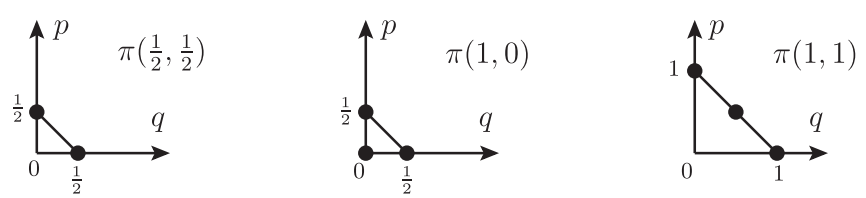

FIG. 1. The lowest order $\pi(p, q)$ representations of $\mathrm{SO}(4,1)$ in terms of $\mathrm{SO}(4) \simeq \mathrm{SU}(2) \otimes \mathrm{SU}(2)$ labels. The dots indicate the $\left(\ell_{1}, \ell_{2}\right)$ values within each representation.

$d(p, q)=\frac{1}{6}(2 q+1)(2 p+3)(p+q+2)(p-q+1)$.

The range of the highest weights $\left(\ell_{1}, \ell_{2}\right)$ for the lowest order representations $\pi_{p, q}$ is depicted in Fig. 1 in terms of the allowed $\left(\ell_{1}, \ell_{2}\right)$ values. From the formula (B10), we find that they have, respectively, the dimensions $d\left(\frac{1}{2}, \frac{1}{2}\right)=4, d(1,0)=5$, and $d(1,1)=10$, reflecting the decomposition in terms of their restriction to $\mathrm{SO}(4)$ : $\pi\left(\frac{1}{2}, \frac{1}{2}\right) \rightarrow D\left(\frac{1}{2}, 0\right) \oplus D\left(0, \frac{1}{2}\right), \pi(1,0) \rightarrow D\left(\frac{1}{2}, 0\right) \oplus D\left(0, \frac{1}{2}\right) \oplus$ $D(0,0), \pi(1,1) \rightarrow D(1,0) \oplus D(0,1) \oplus D\left(\frac{1}{2}, \frac{1}{2}\right)$.

The matrices that form the $\pi_{p, q}$ representation can be considered to be obtained by Wick rotation from the $\mathrm{SO}(5)$ matrices derived in [20]. To better characterize this definition, consider first the defining five-dimensional representation of $\mathrm{SO}(4,1)$ matrices. They are the matrices preserving the bilinear form given by the five-dimensional Lorentzian metric $\eta^{(5)} \equiv(+,-,-,-,-)$, so that the generators are matrices $\mathcal{M}_{A B}$ such that $\mathcal{M}_{A B}^{T}=-\eta^{(5)} \mathcal{M}_{A B} \eta^{(5)}$, and are related with the $\mathfrak{g} \mathfrak{o}(5)$ (skew-symmetric) matrices $\tilde{\mathcal{M}}_{A B}$ by $\tilde{\mathcal{M}}_{A B}=-\eta_{5} \mathcal{M}_{A B}$. We can set then $\left(\mathcal{M}_{A B}\right)_{K L}=$ $\eta_{A K}^{(5)} \delta_{B L}-\eta_{B K}^{(5)} \delta_{A L}$ and $\left(\tilde{\mathcal{M}}_{A B}\right)_{K L}=-\left(\delta_{A K} \delta_{B L}-\delta_{B K} \delta_{A L}\right)$, and the $\mathfrak{s} \mathfrak{v}(4,1)$ commutation rules $\left[\mathcal{M}_{A B}, \mathcal{M}_{C D}\right]=$ $\eta_{A D} \mathcal{M}_{B C}+\eta_{B C} \mathcal{M}_{A D}-\eta_{A C} \mathcal{M}_{B D}-\eta_{B D} \mathcal{M}_{A C}$, as well as the $\mathfrak{g} \mathfrak{v}(5)$ ones for $\tilde{\mathcal{M}}_{A B}$, are satisfied. On passing from $\mathfrak{S o}(5)$ to $\mathfrak{S o}(4,1)$ matrices, one can easily see that the matrices $\mathcal{M}_{0 A}$ become symmetric (and Hermitian), while the remaining ones stay skew symmetric (and antiHermitian). In particular, if $\tilde{\lambda}$ are the (imaginary) eigenvalues of the $\tilde{\mathcal{M}}_{A B}$, from the spectral theorem, the effect of the map on the eigenvalues is $\tilde{\lambda} \rightarrow \lambda=i \tilde{\lambda}$ for the matrices $\mathcal{M}_{0 A}$, while for the remaining $\mathcal{M}_{i A}(i=1,2,3 ; A \neq 0)$ they stay the same. With this in mind, we can generalize this definition of Wick rotation to representations of any dimensions: one can always choose the matrix generators $\mathcal{M}_{0 A}$ to be Hermitian and the $\mathcal{M}_{i A}(A \neq 0)$ anti-Hermitian, and, when going from $\mathrm{SO}(5)$ to $\mathrm{SO}(4,1)$ matrix generators, the eigenvalues of the $\mathcal{M}_{0 A}\left(\equiv\left\{\pi_{p, q}\left(\hat{v}_{0}\right), \pi_{p, q}\left(\hat{S}_{0 i}\right)\right\}\right)$ components acquire an extra $i$ factor. ${ }^{8}$

\footnotetext{
${ }^{8}$ One can check explicitly that this is indeed true for the examples in this paper.
}

We can then use almost the same argument of [21] to establish the relation between the matrices $\mathcal{M}_{\mu 4}=$ $\pi_{p, q}\left(\hat{v}_{\mu}\right)$ and their characteristic polynomial. We observe first that each $\mathrm{SO}(5)$ matrix $\tilde{\mathcal{M}}_{A B}$ is unitary equivalent to a member of a triplet of generators forming angular momentum algebra. It thus follows, considering also the discussion above, that $\tilde{\mathcal{M}}_{A B}$ is equivalent to a direct sum of diagonal block matrices, each one with elements im with $m=j, j-1, \quad,-j$ for $p \geq j \geq 0$. It follows from the Cailey-Hamilton theorem applied to the matrices $\tilde{\mathcal{M}}_{\mu 4}$, and the above considerations, that in the irrep $\pi_{p, q}$ the generators $\hat{v}_{\mu}$ satisfy the minimal characteristic equation,

$$
\prod_{m=-p}^{p}\left(\hat{v}_{\mu}-\left(\delta_{\mu 0}+i \sum_{i} \delta_{\mu i}\right) m \mathbb{1}\right)=0 .
$$

\section{APPENDIX C: SOME PROPERTIES OF THE CORRELATION FUNCTION}

\section{Ward identities}

Under the transformation (29), the action (A3) $S=$ $\int \omega(l)$ varies as

$$
\begin{aligned}
\delta S & =\int\langle\delta X, Y\rangle=\int\left\langle f_{a b}{ }^{c} \xi^{b} l_{c} \tilde{e}^{a}, Y^{d} e_{d}\right\rangle=\int f_{a b}{ }^{c} \xi^{b} l_{c} Y^{a} \\
& =-\int \xi^{a} f_{a b}{ }^{c} Y^{b} l_{c}=-\int \xi^{a} d l_{a}=-\int \xi^{a} \partial_{t} l_{a} d t,
\end{aligned}
$$

where we used Eq. (A6). Notice also that the variation of $l_{a}$ (29) is generated by the coadjoint action of the vector $\xi \in \mathfrak{g}$,

$$
\begin{aligned}
\left\langle\operatorname{ad}^{*}(\xi) X, e_{a}\right\rangle & =\xi^{b}\left\langle X,\left[e_{a}, e_{b}\right]\right\rangle=f_{a b}^{c} \xi^{b}\left\langle X, e_{c}\right\rangle \\
& =f_{a b} \xi^{b} l_{c}=\delta l_{a} .
\end{aligned}
$$

We exploit now the invariance of the functional integral

$$
\delta \int \mathcal{D}(l) \prod_{i} e^{i S(l)} l_{a_{1}}\left(t_{1}\right) \cdots l_{a_{n}}\left(t_{n}\right)=0
$$

under this transformation (under the coadjoint action (C2) the measure is invariant). The term $e^{i S\left(l_{a}\right)}$ changes as in (1), i.e.,

$$
\delta e^{i S}=-i e^{i S} \int \xi^{a} \partial_{t} l_{a}(t) d t
$$

while the terms $l^{a_{i}}\left(t_{i}\right)$ change as

$$
\delta l_{a_{i}}\left(t_{i}\right)=f_{a_{i} b}{ }^{c} \xi^{b} l_{c}\left(t_{i}\right)=\int f_{a_{i} b} \xi^{b} l_{c}(t) \delta\left(t-t_{i}\right) d t .
$$


Then, we get from (C3),

$$
\int D(l) e^{i S} \int d t\left(-i \xi^{a} \partial_{t} l_{a}(t) l_{a_{1}}\left(t_{1}\right) \cdots l_{a_{n}}\left(t_{n}\right)+\sum_{k} f_{a_{k} b}^{c} \xi^{b} \delta\left(t-t_{k}\right) l_{c}(t) l_{a_{1}}\left(t_{1}\right) \cdots \bar{l}_{a_{k}}\left(t_{k}\right) \cdots l_{a_{n}}\left(t_{n}\right)\right)=0
$$

where $\bar{l}_{a_{1}}\left(t_{k}\right)$ means that this term is missing from the product. From these relations, we obtain the Ward identities (30).

\section{2. n-point correlation function}

One can show that the n-point correlation function is given by the time ordered product of the field operators as

$$
\left\langle i\left|\hat{l}_{a_{1}}\left(t_{1}\right) \cdots \hat{l}_{a_{n}}\left(t_{n}\right)\right| j\right\rangle=\sum_{\left\{i_{1} \cdots i_{n}\right\}} \prod_{k=1}^{n-1} \theta\left(t_{i_{k}}-t_{i_{k+1}}\right)\left\langle i\left|\hat{l}_{a_{i_{1}}} \ldots \hat{l}_{a_{i_{n}}}\right| j\right\rangle
$$

where on the r.h.s. $\hat{l}_{a}$ are matrix operators for a specific representation of $\mathfrak{g} \mathfrak{v}(4,1)$, and summation is over all permutations $\left\{i_{1} \cdots i_{n}\right\}$ of the numbers $(1 \cdots n)(\theta(x)$ is the Heaviside step function). Indeed, the Ward identities (30) are satisfied, as can be shown explicitly by deriving Eq. (C7). For instance, for $n=3$, denoting $\delta_{i j}=\delta\left(t_{i}-t_{j}\right)$ and $\theta_{i j}=\theta\left(t_{i}-t_{j}\right)$, and using that $\partial / \partial x \theta( \pm x)= \pm \delta( \pm x)$

$$
\begin{aligned}
\partial_{t_{1}}\left\langle i\left|\hat{l}_{a_{1}}\left(t_{1}\right) \hat{l}_{a_{2}}\left(t_{2}\right) \hat{l}_{a_{3}}\left(t_{3}\right)\right| j\right\rangle= & \delta_{12}\left(\theta_{23}\left\langle i\left|\left[\hat{l}_{a_{1}}, \hat{l}_{a_{2}}\right] \hat{l}_{a_{3}}\right| j\right\rangle+\theta_{32}\left\langle i\left|\hat{l}_{a_{3}}\left[\hat{l}_{a_{1}}, \hat{l}_{a_{2}}\right]\right| j\right\rangle\right) \\
& +\delta_{13}\left(\theta_{32}\left\langle i\left|\left[\hat{l}_{a_{1}}, \hat{l}_{a_{3}}\right] \hat{l}_{a_{2}}\right| j\right\rangle+\theta_{23}\left\langle i\left|\hat{l}_{a_{2}}\left[\hat{l}_{a_{1}}, \hat{l}_{a_{3}}\right]\right| j\right\rangle\right) \\
= & i f_{a_{1} a_{2}}^{b} \delta_{12}\left\langle i\left|\hat{l}_{b}\left(t_{2}\right) \hat{l}_{a_{3}}\left(t_{3}\right)\right| j\right\rangle+i f_{a_{1} a_{3}}^{b} \delta_{13}\left\langle i\left|\hat{l}_{a_{2}}\left(t_{2}\right) \hat{l}_{b}\left(t_{3}\right)\right| j\right\rangle,
\end{aligned}
$$

where we used the ETC (34), and in general (30) are verified. One can also check that the BJL limit is satisfied by (C7). Indeed (see [36]), using the integral representation of the step function,

$$
\theta(t)=\frac{i}{2 \pi} \int d \alpha e^{-i \alpha t} \frac{1}{\alpha+i \epsilon}
$$

and (C7) for $n=2$, Eq. (31) can be rewritten as

$$
\begin{aligned}
& \frac{i}{2 \pi} \lim _{p_{0} \rightarrow \infty} p_{0} \int d t \int \frac{d \alpha}{\alpha+i \epsilon} e^{i p_{0}\left(t-t_{1}\right)}\left(e^{-i \alpha\left(t-t_{1}\right)}\left\langle i\left|\hat{l}_{a} \hat{l}_{a_{1}}\right| j\right\rangle+e^{i \alpha\left(t-t_{1}\right)}\left\langle i\left|\hat{l}_{a_{1}} \hat{l}_{a}\right| j\right\rangle\right) \\
& \quad=\frac{i}{2 \pi} \lim _{p_{0} \rightarrow \infty} p_{0} \int d t \int d p_{0}^{\prime} e^{i p_{0}^{\prime}\left(t-t_{1}\right)}\left(\frac{1}{p_{0}-p_{0}^{\prime}+i \epsilon}\left\langle i\left|\hat{l}_{a} \hat{l}_{a_{1}}\right| j\right\rangle-\frac{1}{p_{0}-p_{0}^{\prime}-i \epsilon}\left\langle i\left|\hat{l}_{a_{1}} \hat{l}_{a}\right| j\right\rangle\right) .
\end{aligned}
$$

Taking the limit for $p_{0} \rightarrow \infty$, it becomes

$$
i \int d t \delta\left(t-t_{1}\right)\left\langle i\left|\left[\hat{l}_{a}, \hat{l}_{a_{1}}\right]\right| j\right\rangle=i\left\langle i\left|\left[\hat{l}_{a}, \hat{l}_{a_{1}}\right]\left(t_{1}\right)\right| j\right\rangle
$$

Finally, we obtain the correlation function,

$$
\begin{aligned}
\left\langle i\left|\exp \left(i p_{\mu} \int_{0}^{L} \hat{v}^{\mu}(t) d t\right)\right| j\right\rangle & =\sum_{n=0}^{\infty} \frac{i^{n}}{n !} p_{\mu_{1}} p_{\mu_{2}} \cdots p_{\mu_{n}} \prod_{k=1}^{n} \int_{0}^{L} d t_{k}\left\langle i\left|\hat{v}^{\mu_{1}}\left(t_{1}\right) \hat{v}^{\mu_{2}}\left(t_{2}\right) \cdots \hat{v}^{\mu_{n}}\left(t_{n}\right)\right| j\right\rangle \\
& =\sum_{n=0}^{\infty} \frac{i^{n}}{n !} p_{\mu_{1}} p_{\mu_{2}} \cdots p_{\mu_{n}} \prod_{k=1}^{n} \int_{0}^{L} d t_{k} \prod_{m=1}^{n-1} \theta\left(t_{m}-t_{m+1}\right) \sum_{i_{1} \cdots i_{n}}\left\langle i\left|\hat{v}^{\mu_{i_{1}}} \cdots \hat{v}^{\mu_{i_{n}}}\right| j\right\rangle \\
& =\sum_{n=0}^{\infty} \frac{i^{n}}{n !} L^{n} p_{\mu_{1}} p_{\mu_{2}} \cdots p_{\mu_{n}}\left\langle i\left|\hat{v}^{\mu_{i_{1}}} \cdots \hat{v}^{\mu_{i_{n}}}\right| j\right\rangle \\
& =\langle i|\exp (i L p \cdot \hat{v})| j\rangle
\end{aligned}
$$


where we used Eq. (C7) relation,

$$
\prod_{k=1}^{n} \int_{0}^{L} d t_{k} \prod_{m=1}^{n-1} \theta\left(t_{m}-t_{m+1}\right)=\frac{L^{n}}{n !}
$$

and the fact that the indices $\mu, \nu, \rho, \ldots$ are saturated by the symmetric terms $p_{\mu} p_{\nu} p_{\rho} \cdots$, so that the sum over permutations of indices $i_{1}, i_{2}, \ldots i_{n}$ gives another $n$ ! factor. On the r.h.s. of $(\mathrm{C} 11)$, the $\hat{v}^{\mu}$ are matrices $\pi\left(\hat{v}^{\mu}\right)$ in the given $\mathfrak{s o}(4,1)$ representation.

\section{APPENDIX D: MATRIX REPRESENTATION FOR THE SPIN-1 SECTOR}

\section{The relation between $\ell=1$ representation of $S O(4,1)$ and the DKP algebra}

For $k=1 \mathrm{Eq}$. (41), $\beta_{\mu}=-i \hat{v}_{\mu}$ gives

$$
\beta_{\mu}^{3}=\eta_{\mu \mu} \beta_{\mu} .
$$

Plugging this into (40), we obtain, for $\rho=\nu$ and $\mu \neq \nu$,

$$
\beta_{\mu} \beta_{\nu}^{2}-2 \beta_{\nu} \beta_{\mu} \beta_{\nu}+\beta_{\nu}^{2} \beta_{\mu}=\beta_{\mu} \eta_{\nu \nu} .
$$

Multiplying from the right by $\beta_{\nu}$ and using (D1) follow

$$
\beta_{\nu}^{2} \beta_{\mu} \beta_{\nu}=2 \beta_{\nu} \beta_{\mu} \beta_{\nu}^{2} .
$$

Multiplying from the right and left by $\beta_{\nu}$ follow, respectively, $\left.\begin{array}{l}\beta_{\nu}^{2} \beta_{\mu} \beta_{\nu}^{2}=2 \beta_{\nu} \beta_{\mu} \beta_{\nu} \eta_{\nu \nu} \\ \beta_{\nu} \beta_{\mu} \beta_{\nu} \eta_{\nu \nu}=2 \beta_{\nu}^{2} \beta_{\mu} \beta_{\nu}^{2}\end{array}\right\} \Rightarrow \beta_{\nu} \beta_{\mu} \beta_{\nu}=0 \quad \mu \neq \nu$,

so that (D2) becomes

$$
\beta_{\mu} \beta_{\nu}^{2}+\beta_{\nu}^{2} \beta_{\mu}=\beta_{\mu} \eta_{\nu \nu} \quad \mu \neq \nu .
$$

Now, we take $\mu \neq \nu \neq \rho$ in (40), multiply from the left twice by $\beta_{\nu}$ and use (D3) and (D1) to get

$$
\beta_{\nu} \beta_{\mu} \beta_{\rho} \eta_{\nu \nu}+\beta_{\nu}^{2} \beta_{\rho} \beta_{\mu} \beta_{\nu}=0 \quad \mu \neq \nu \neq \rho .
$$

Finally, we use relation (D4) and obtain

$$
\beta_{\nu} \beta_{\mu} \beta_{\rho}+\beta_{\rho} \beta_{\mu} \beta_{\nu}=0 \quad \mu \neq \nu \neq \rho .
$$

Relations (D1), (D4), and (D5) define the DKP (DuffinKemmer-Petiau) algebra,

$$
\beta_{\mu} \beta_{\rho} \beta_{\nu}+\beta_{\nu} \beta_{\rho} \beta_{\mu}=\beta_{\mu} \eta_{\nu \rho}+\beta_{\nu} \eta_{\mu \rho}
$$

\section{Five-dimensional representation of DKP matrices for spin-0}

The DKP matrices (44) admit a five-dimensional irreducible representation that carries the degrees of freedom of a spin-0 field theory. We here report an explicit expression for the matrices in this representation [14]. These are given as

$$
\beta_{0}=\left(\begin{array}{ccccc}
0 & 0 & 0 & 0 & 1 \\
0 & 0 & 0 & 0 & 0 \\
0 & 0 & 0 & 0 & 0 \\
0 & 0 & 0 & 0 & 0 \\
1 & 0 & 0 & 0 & 0
\end{array}\right), \quad \beta_{1}=\left(\begin{array}{ccccc}
0 & 0 & 0 & 0 & 0 \\
0 & 0 & 0 & 0 & 1 \\
0 & 0 & 0 & 0 & 0 \\
0 & 0 & 0 & 0 & 0 \\
0 & -1 & 0 & 0 & 0
\end{array}\right), \quad \beta_{2}=\left(\begin{array}{ccccc}
0 & 0 & 0 & 0 & 0 \\
0 & 0 & 0 & 0 & 0 \\
0 & 0 & 0 & 0 & 1 \\
0 & 0 & 0 & 0 & 0 \\
0 & 0 & -1 & 0 & 0
\end{array}\right), \quad \beta_{3}=\left(\begin{array}{ccccc}
0 & 0 & 0 & 0 & 0 \\
0 & 0 & 0 & 0 & 0 \\
0 & 0 & 0 & 0 & 0 \\
0 & 0 & 0 & 0 & 1 \\
0 & 0 & 0 & -1 & 0
\end{array}\right) .
$$

Notice that with this definition the spin- $0 \beta$ matrices coincide with the defining representation for the generators corresponding to the "momentum sector" of $S O(4,1)$, i.e., $\beta_{\mu} \equiv M_{\mu 4}$, where $\left(M_{A B}\right)_{K L}:=\eta_{A K} \delta_{K L}-\eta_{B K} \delta_{A L}$ (where here $\left.\eta_{A B}=\operatorname{diag}(1,-1,-1,-1,-1)\right)$. In this representation, the projection of the field is

$$
\psi=\left(\begin{array}{c}
\pi_{0} \\
\pi_{1} \\
\pi_{2} \\
\pi_{3} \\
\varphi
\end{array}\right) \longrightarrow \phi=\mathcal{P} \psi=\left(\begin{array}{l}
0 \\
0 \\
0 \\
0 \\
\varphi
\end{array}\right)
$$




\section{Ten-dimensional representation of DKP matrices for spin-1}

The ten-dimensional representations for the $\beta$ matrices can be given as [22]

$$
\beta_{0}=\left(\begin{array}{cccccccccc}
0 & 0 & 0 & 0 & 0 & 0 & 1 & 0 & 0 & 0 \\
0 & 0 & 0 & 0 & 0 & 0 & 0 & 1 & 0 & 0 \\
0 & 0 & 0 & 0 & 0 & 0 & 0 & 0 & 1 & 0 \\
0 & 0 & 0 & 0 & 0 & 0 & 0 & 0 & 0 & 0 \\
0 & 0 & 0 & 0 & 0 & 0 & 0 & 0 & 0 & 0 \\
0 & 0 & 0 & 0 & 0 & 0 & 0 & 0 & 0 & 0 \\
1 & 0 & 0 & 0 & 0 & 0 & 0 & 0 & 0 & 0 \\
0 & 1 & 0 & 0 & 0 & 0 & 0 & 0 & 0 & 0 \\
0 & 0 & 1 & 0 & 0 & 0 & 0 & 0 & 0 & 0 \\
0 & 0 & 0 & 0 & 0 & 0 & 0 & 0 & 0 & 0
\end{array}\right), \quad \beta_{1}=\left(\begin{array}{ccccccccccccccc}
0 & 0 & 0 & 0 & 0 & 0 & 0 & 0 & 0 & 1 \\
0 & 0 & 0 & 0 & 0 & 0 & 0 & 0 & 0 & 0 \\
0 & 0 & 0 & 0 & 0 & 0 & 0 & 0 & 0 & 0 \\
0 & 0 & 0 & 0 & 0 & 0 & 0 & 0 & 0 & 0 \\
0 & 0 & 0 & 0 & 0 & 0 & 0 & 0 & 1 & 0 \\
0 & 0 & 0 & 0 & 0 & 0 & 0 & -1 & 0 & 0 \\
0 & 0 & 0 & 0 & 0 & 0 & 0 & 0 & 0 & 0 \\
0 & 0 & 0 & 0 & 0 & 0 & 0 & 0 & 0 \\
0 & 0 & 0 & 0 & 0 & 0 & 0 & 0 & 0 & 0 \\
0 & 0 & 0 & 0 & 0 & 0 & 0 & 0 & 1 & 0 \\
0 & 0 & 0 & 0 & 0 & 0 & 0 & 0 & 0 & 0 \\
0 & 0 & 0 & 0 & 0 & 0 & 1 & 0 & 0 & 0 \\
0 & 0 & 0 & 0 & 0 & -1 & 0 & 0 & 0 & 0 \\
0 & 0 & 0 & -1 & 0 & 0 & 0 & 0 & 0 \\
-1 & 0 & 0 & 0 & 0 & 0 & 0 & 0 & 0 & 0
\end{array}\right), \quad \beta_{3}=\left(\begin{array}{ccccccccccccccc}
0 & 0 & 0 & 0 & 0 & 0 & 0 & 0 & 0 & 0 \\
0 & 0 & 0 & 0 & 0 & 0 & 0 & 0 & 0 & 0 \\
0 & 0 & 0 & 0 & 0 & 0 & 0 & 0 & 0 & 1 \\
0 & 0 & 0 & 0 & 0 & 0 & 0 & 1 & 0 & 0 \\
0 & 0 & 0 & 0 & 0 & 0 & 1 & 0 & 0 & 0 \\
0 & 0 & 0 & 0 & 0 & 0 & 0 & 0 & 0 & 0 \\
0 & 0 & 0 & 0 & 0 & 0 & 0 & 0 & 0 & 0 \\
0 & -1 & 0 & 0 & 0 & 0 & 0 & 0 & 0 & 0 & 0 \\
0 & 0 & 0 & 0 & 1 & 0 & 0 & 0 & 0 & 0 \\
0 & 0 & 0 & -1 & 0 & 0 & 0 & 0 & 0 & 0 \\
0 & 0 & 0 & 0 & 0 & 0 & 0 & 0 & 0 & 0 \\
0 & 0 & -1 & 0 & 0 & 0 & 0 & 0 & 0 & 0
\end{array}\right) .
$$

With these representations, the field $\psi$ is projected as

$$
\psi=\left(\begin{array}{c}
F_{23} \\
F_{31} \\
F_{12} \\
F_{01} \\
F_{02} \\
F_{03} \\
A_{1} \\
A_{2} \\
A_{3} \\
A_{0}
\end{array}\right) \longrightarrow \mathcal{A}_{\mu}=\mathcal{R}_{\mu} \psi=\left(\begin{array}{c}
0 \\
0 \\
0 \\
0 \\
0 \\
0 \\
0 \\
0 \\
0 \\
A_{\mu}
\end{array}\right),
$$

while

$$
\overline{\mathcal{A}}_{\mu}=-\psi^{\dagger} \eta_{0} \mathcal{R}_{\mu}^{\dagger}=\left(\begin{array}{llllllllll}
0 & 0 & 0 & 0 & 0 & 0 & 0 & 0 & 0 & \eta_{\mu \mu} \mathcal{A}_{\mu}
\end{array}\right),
$$

so that the scalar product is given by

$$
\sum_{\mu} \overline{\mathcal{A}}_{\mu} \mathcal{A}_{\mu}=\mathcal{A}_{\mu} \mathcal{A}^{\mu}=\eta^{\mu \nu} A_{\mu} A_{\nu}=A_{\mu} A^{\mu}
$$


[1] A. Frydryszak, Lagrangian models of the particles with spin: The first seventy years, From Field Theory to Quantum Groups, edited by B. Jancewicz and J. Sobczyk (World Scientific, 1996), pp. 151-172, and University of Wroclaw, ITP-UWr-96-0901 (1996), p. 25.

[2] A. P. Balachandran, G. Marmo, B. S. Skagerstam, and A. Stern, Spinning particles in general relativity, Phys. Lett. 89B, 199 (1980).

[3] A. P. Balachandran, G. Marmo, B.-S. Skagerstam, and A. Stern, Gauge Theories and Fibre Bundles-Applications to Particle Dynamics, Lecture Notes in Physics Vol. 188 (Springer, New York, 1983); available also as Lect. Notes Phys. 188, 1 (1983).

[4] P. de Sousa Gerbert, On spin and (quantum) gravity in $(2+1)$-dimensions, Nucl. Phys. B346, 440 (1990).

[5] L. Freidel, J. Kowalski-Glikman, and A. Starodubtsev, Particles as Wilson lines of gravitational field, Phys. Rev. D 74, 084002 (2006).

[6] T. Rempel and L. Freidel, A classical and spinorial description of the relativistic spinning particle, arXiv: 1612.00551.

[7] A. Y. Alekseev and S. L. Shatashvili, Propagator for the relativistic spinning particle via functional integral over trajectories, Mod. Phys. Lett. A 03, 1551 (1988).

[8] P. Orland, Bosonic path integrals for four-dimensional Dirac particles, Int. J. Mod. Phys. A 04, 3615 (1989).

[9] A. M. Polyakov, Fermi-Bose transmutations induced by gauge fields, Mod. Phys. Lett. A 03, 325 (1988).

[10] L. Freidel, J. Kowalski-Glikman, R. G. Leigh, and D. Minic, The theory of metaparticles, Phys. Rev. D 99, 066011 (2019).

[11] R. J. Duffin, On the characteristic matrices of covariant systems, Phys. Rev. 54, 1114 (1938).

[12] N. Kemmer, The particle aspect of meson theory, Proc. R. Soc. A 173, 91 (1939).

[13] G. Petiau, Ph.D. thesis, University of Paris, 1936, published in Acad. R. Belg., Classe Sci., Mem. in 8o 16, No. 2 (1936).

[14] J. T. Lunardi, B. M. Pimentel, R. G. Teixeira, and J. S. Valverde, Remarks on Duffin-Kemmer-Petiau theory and gauge invariance, Phys. Lett. A 268, 165 (2000).

[15] S. Weinberg, The Quantum Theory of Fields. Vol. 1: Foundations (Cambridge University Press, Cambridge, United Kingdom, 1995).

[16] A. Alekseev, L. D. Faddeev, and S. L. Shatashvili, Quantization of symplectic orbits of compact Lie groups by means of the functional integral, J. Geom. Phys. 5, 391 (1988).

[17] J. Dixmier, Représentations intérables du groupe de De Sitter, Bull Soc. Math. (France) 89, 9 (1961).

[18] L. H. Thomas, On unitary representations of the group of de Sitter space, Ann. Math. 42, 113 (1941).
[19] T. D. Newton, A note on the representations of the de Sitter group, Ann. Math. 51, 730 (1950).

[20] N. Kemmer, D. L. Pursey, and S. A. Williams, Irreducible representations of the five-dimensional rotation group. I, J. Math. Phys. (N.Y.) 9, 1224 (1968).

[21] E. Fischbach, J. D. Louck, M. M. Nieto, and C. K. Scott, The Lie algebra so(n) and the Duffin-Kemmer-Petiau ring, J. Math. Phys. (N.Y.) 15, 60 (1974).

[22] H. Umezawa, Quantum Field Theory (North-Holland Publishing Co., Amsterdam, 1956).

[23] J. Beltran, B. M. Pimentel, and D. E. Soto, Scattering process in the Scalar Duffin-Kemmer-Petiau gauge theory, J. Phys. Conf. Ser. 706, 052019 (2016).

[24] J. Lukierski, H. Ruegg, A. Nowicki, and V. N. Tolstoi, $Q$ deformation of Poincare algebra, Phys. Lett. B 264, 331 (1991).

[25] J. Lukierski, A. Nowicki, and H. Ruegg, New quantum Poincare algebra and k deformed field theory, Phys. Lett. B 293, 344 (1992).

[26] S. Majid and H. Ruegg, Bicrossproduct structure of kappa Poincare group and noncommutative geometry, Phys. Lett. B 334, 348 (1994).

[27] J. Kowalski-Glikman, A short introduction to $\kappa$-deformation, Int. J. Mod. Phys. A 32, 1730026 (2017).

[28] A. Nowicki, E. Sorace, and M. Tarlini, The quantum deformed Dirac equation from the kappa Poincare algebra, Phys. Lett. B 302, 419 (1993).

[29] P. Kosinski, P. Maslanka, J. Lukierski, and A. Sitarz, Towards kappa-deformed $D=4$ relativistic field theory, Czech. J. Phys. 48, 1407 (1998).

[30] A. Agostini, G. Amelino-Camelia, and M. Arzano, Dirac spinors for doubly special relativity and kappa Minkowski noncummutative space-time, Classical Quantum Gravity 21, 2179 (2004).

[31] A. Alekseev and S. L. Shatashvili, Path integral quantization of the coadjoint orbits of the Virasoro group and 2D gravity, Nucl. Phys. B323, 719 (1989).

[32] A. A. Kirillov, Lectures on the Orbit Method, Graduate Studies in Mathematics Vol. 64 (American Mathematical Society, Providence, 2004).

[33] N. M.J. Woodhouse, Geometric Quantization, Oxford Mathematical Monographs (Clarendon, New York, 1992), p. 307.

[34] B. C. Hall, Quantum Theory for Mathematicians (SpringerVerlag, New York, 2013).

[35] N. T. Evans, Discrete series for the universal covering group of the $3+2$ de Sitter group, J. Math. Phys. (N.Y.) 8, 170 (1967).

[36] R. Jackiw, in Current Algebras and Anomalies (Princeton University Press, Princeton, NJ, 1985). 